\title{
Afterbody vortices of axisymmetric cylinders with a slanted base
}

\author{
D. S. Bulathsinghala ${ }^{1} \cdot$ R. Jackson ${ }^{1} \cdot$ Z. Wang $^{1} \cdot$ I. Gursul $^{1} \odot$
}

Received: 20 December 2016 / Accepted: 7 April 2017 / Published online: 22 April 2017

(c) The Author(s) 2017. This article is an open access publication

\begin{abstract}
Experiments have been undertaken to study the formation of afterbody vortex flows from cylindrical bodies with a slanted base, whose upsweep angle was varied between $24^{\circ}$ and $32^{\circ}$. Vortex roll-up is mostly completed in the first half of the upswept section, where the vortex causes largest suction on the surface. Towards the trailing-edge the vortices become more axisymmetric and stronger with increasing upsweep angle. Although there is some delay in vortex rollup at lower Reynolds number, the main features of the vortex flow are similar to those at higher Reynolds number. The strength of the vortices at the trailing-edge was proportional to the time-averaged drag coefficient, which increased by nearly $50 \%$ in the range of upsweep angles tested. The vortex was more coherent with reduced meandering and a smaller core radius towards the trailing-edge. This reduction in meandering along the streamwise direction had not been observed previously with other external vortex flows in aerodynamics. Proper Orthogonal Decomposition revealed that the helical displacement mode with azimuthal wavenumber $m=1$ was the dominant mode towards the trailing-edge, suggesting that the afterbody vortices bear much similarity with the more widely studied wing tip vortices and delta wing vortices. The instantaneous vortex pair exhibits time-dependent asymmetry; however, there is virtually no correlation between the displacements of the vortex centers.
\end{abstract}

\section{List of symbols}

$a_{m} \quad$ Meandering amplitude

$C_{D} \quad$ Drag coefficient

I. Gursul

i.a.gursul@bath.ac.uk

1 Department of Mechanical Engineering, University of Bath, Bath BA2 7AY, UK
$C_{\mathrm{p}} \quad$ Pressure coefficient

$D \quad$ Model fuselage diameter

$L \quad$ Afterbody length

$m \quad$ Azimuthal wavenumber

$N \quad$ Number of instantaneous PIV snapshots

$P \quad$ Probability of instantaneous vortex core location

$R \quad$ Vortex spatial correlation coefficient

$R e_{D} \quad$ Reynolds number based on diameter

$r_{\text {core }}$ Time-averaged vortex core radius

$U_{\infty} \quad$ Freestream velocity

$x \quad$ Streamwise coordinate

$y \quad$ Vertical coordinate in crossflow plane

$y_{c} \quad$ Vertical coordinate of time-averaged vortex core location

$y_{i} \quad$ Vertical coordinate of instantaneous vortex core location

$z \quad$ Horizontal coordinate in crossflow plane

$z_{c} \quad$ Horizontal coordinate of time-averaged vortex core location

$z_{i} \quad$ Horizontal coordinate of instantaneous vortex core location

$\Gamma \quad$ Circulation

$\Phi \quad$ Upsweep angle

$\omega \quad$ Vorticity

\section{Introduction}

Military transport aircraft typically have pronounced upswept fuselage afterbodies, which are required by design to facilitate the rear loading of cargo. The presence of this afterbody shape results in the formation of two counterrotating vortices which exist in the vicinity of the upswept fuselage (Epstein et al. 1994). This vortex pair creates a low pressure region, which results in a larger drag coefficient 
when compared to conventional passenger aircraft with shallower upsweeps (Bearman 1980). The vortex pair generates a strong upwash, which may interfere with airdrop missions (Bury et al. 2013).

The influence of upsweep angle upon the drag coefficient was initially explored during an experimental campaign at General Motors research laboratories, with an interest in examining the flow field downstream of the rear roof slant of hatchback cars (Morel 1980). The experiments were performed on cylindrical models with slanted bases at zero incidence, and it was concluded that the upsweep angle $\Phi$ (see the definition in Fig. 1a) of the afterbody had a significant effect on the flow field and drag coefficient. There exists a critical upsweep angle at which the flow regime changes from a counter-rotating vortex pair structure (which is of interest to the current study) to a separated wake. This critical upsweep angle occurs at around $\Phi=45^{\circ}$ to $50^{\circ}$ (Morel 1980; Bearman 1980) and can be sensitive to the experimental setup. For upsweep angles smaller than the critical angle, the time-averaged drag coefficient increases with increasing upsweep angle. There is a sudden drop in the drag coefficient at the critical upsweep angle. Similar experiments have been performed by Maull (1980), Xia and Bearman (1983) and Britcher and Alcorn (1991), which have used similar slanted base cylindrical models and reported the same trends in the drag coefficient. Epstein et al. (1994) and Peake et al. (1972) claimed the absence of a vortex shedding frequency in the vortex pair regime, and similarities in the structure of the flow field were drawn to delta wings. Epstein et al. (1994) also concluded that the size and shape of the afterbody vortex was only weakly, if at all, dependent upon the Reynolds number. Bury et al. (2013) investigated the near-wake flow field using crossflow measurements and discussed the effects of having open and closed ramp doors on the afterbody vortex flow field. Opening the cargo ramp resulted in the formation of an induced pair of cargo ramp vortices with opposite vorticity compared to the existing afterbody vortex pair.
Fig. 1 a Model parameters; b view of $\Phi=28^{\circ}$ wind tunnel model showing pressure tap locations on starboard side

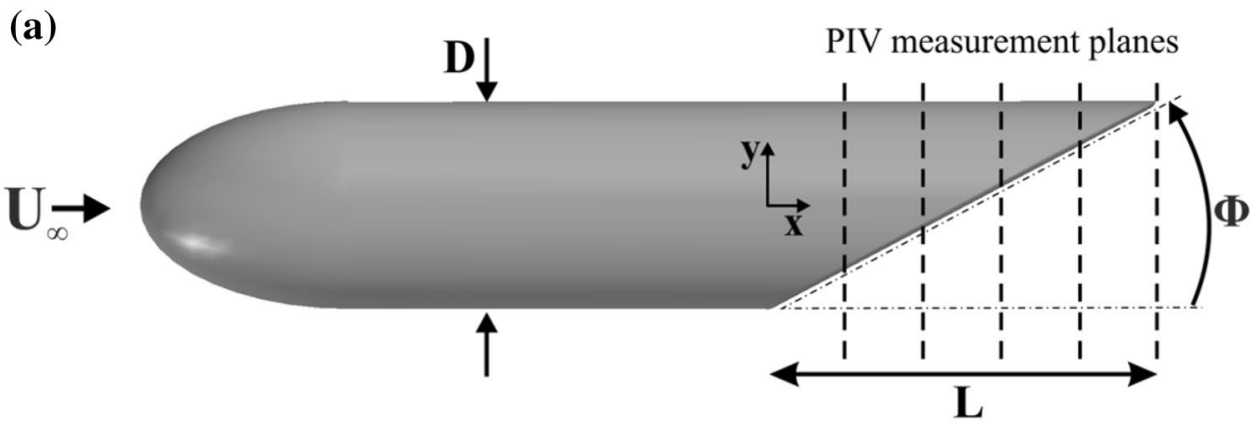

(b)

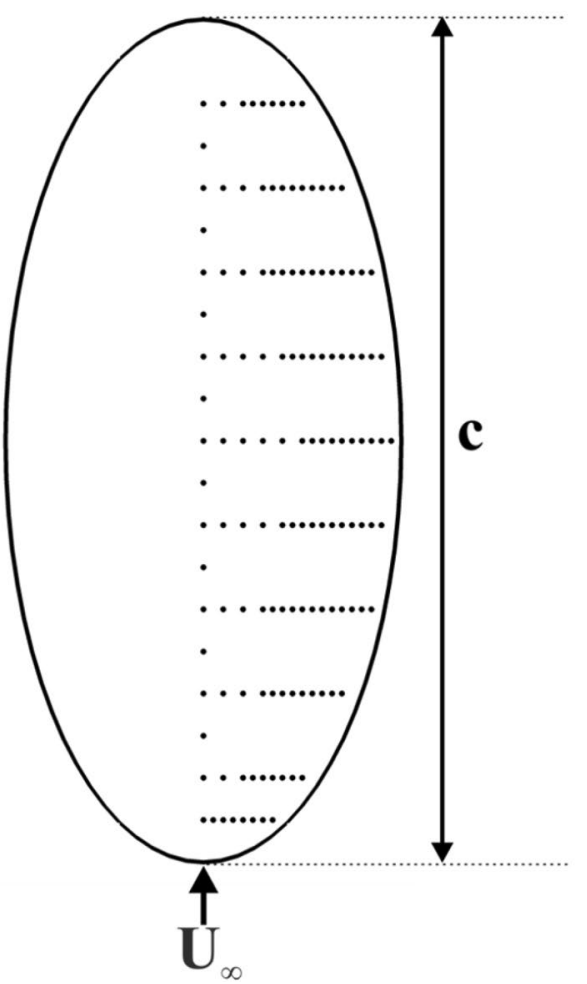


The resulting complex flow field within the cargo bay cavity created a stronger upward flow compared to the closed cargo door configuration.

There have been several experimental campaigns focusing on flow control of afterbody vortices, with the aim of drag reduction. The earliest known example of this was during the development of the Short Belfast aircraft (McCluney and Marshall 1967). A pair of vertical strakes was fitted onto the underside of the afterbody to disrupt the inflow of air and mitigate vortex formation, resulting in drag reductions of $7 \%$. The effectiveness was measured using wind tunnel force measurements and flight tests. The majority of passive flow control techniques employ vortex generators, which promote secondary flow structures that may interact favorably with the afterbody flow field. Calarese et al. (1985) reported drag reduction with the use of vortex generators on a Lockheed Martin C-130 scale model, supported by oil flow visualizations, pressure and force measurements. Wortman (1999) also tested different vortex generator geometries on scale models of Boeing 747 and Lockheed C5 afterbodies reporting drag reductions of 3 and $6 \%$, respectively. Lockheed Martin carried out a combined CFD and flight test program to investigate the effect of microvanes on the afterbody drag on a C-130 (Smith et al. 2013). The microvanes tested consisted of a snug-free design to avoid interference with airdropping missions. The drag reduction potential of these microvanes was realized in terms of fuel savings for the operator. A similar CFD investigation of the potential of finlets and microvanes in producing drag reduction on a Lockheed Martin C-130 was reported by Telli et al. (2016), suggesting total aircraft drag reductions of $4 \%$. There is limited evidence of active flow control applied to this type of flow field, but results by Jackson et al. (2015) highlight the benefits of blowing jets which modify the structure of the afterbody vortices. PIV results revealed that the outboard ejection of momentum from the upswept face of a slanted base cylinder can mitigate the afterbody vortex formation via jet/vortex interactions, suggesting a potential for drag reduction.

The current study is an investigation of afterbody vortex flows in the near-wake of slanted base cylindrical models, and is comprised of drag measurements, surface pressure measurements and 2D crossflow particle image velocimetry (PIV) measurements. The wind tunnel tests are performed on models with a range of upsweep angles that encompass the majority of military and commercial cargo aircraft, which fall within the vortex flow regime. The model used in the water tunnel experiments has an upsweep angle in the mid-range and provides an opportunity to assess the influence of Reynolds number. The cylindrical slanted base models used in this experiment allow for comparisons with studies discussed previously (Morel 1980; Maull 1980; Xia and Bearman 1983; Britcher and Alcorn 1991). In-depth analysis of the instantaneous PIV snapshots, vortex meandering and proper orthogonal decomposition (POD) was carried out to understand the unsteady aspects of the vortex flow.

\section{Experimental techniques}

\subsection{Wind tunnel experimental setup}

The experiments were performed within the closed return wind tunnel at the Department of Mechanical Engineering, University of Bath. The test area of the tunnel has an octagonal cross-section with overall dimensions $2.13 \times 1.52 \times 2.70 \mathrm{~m}$. The freestream turbulence intensity is below $0.4 \%$. The freestream velocity in the working section was monitored using a pitot-static probe mounted within the tunnel connected to a digital manometer. The velocity was set at $U_{\infty}=15 \mathrm{~m} / \mathrm{s}$, which resulted in a Reynolds number (based on model diameter, $D$ ) of $R e_{D}=2 \times 10^{5}$.

\subsubsection{Wind tunnel models and force measurement}

The slanted base cylindrical model used for the experiments is presented in Fig. 1a, in reference with the associated axes. Five models were fabricated with upsweep angles $\Phi=24^{\circ}, 26^{\circ}, 28^{\circ}, 30^{\circ}$ and $32^{\circ}$. The $\Phi=28^{\circ}$ afterbody was equipped with pressure taps on the upswept surface (Fig. 1b). Each cylindrical fuselage section was fabricated using PVC pipe with an external diameter $D=0.20 \mathrm{~m}$. The slants were machined out of the pipe and were covered using 3-mm thick PVC sheets to create each upswept surface. The afterbody length, $L$, varied between 0.45 and $0.32 \mathrm{~m}$, depending upon the upsweep angle of each model. The total model length measured from the nose varied between 1.05 and $0.92 \mathrm{~m}$. The detachable ellipsoidal nose cone was manufactured using solid laser sintering, with the geometry characterized by a 2:1 major to minor axis ratio. A support system transferred the weight of the model to a binocular force balance. Pressure taps passed through a hollow tube within the support structure, which itself was enclosed within a streamlined fairing manufactured from glass fiber. The combined blockage effect of the model and support system was about $2 \%$ of the total working section area.

The binocular force balance consisted of two stress concentration throats where strain gauges were mounted on either side. A full Wheatstone bridge circuit was used to measure the change in strain. Drag data were acquired at a sample frequency of $1 \mathrm{kHz}$ for $10 \mathrm{~s}$ with six repeat measurements per model to obtain accurate readings. The force balance was calibrated for each model separately 
and regular calibration checks were carried out over extended testing periods to account for any drifts within the calibration. The uncertainty in the drag coefficient was estimated to be $2 \%$, using the methods introduced by Moffat (1988). The drag of the support system was measured and subtracted from the total drag to isolate the model drag force.

\subsubsection{Pressure measurements}

The $\Phi=28^{\circ}$ wind tunnel model was chosen for pressure measurements. Its upswept surface was equipped with 134 surface pressure taps (Fig. 1b), each of $1.6 \mathrm{~mm}$ diameter. The taps were distributed on the starboard side of the upsweep, corresponding to the PIV measurements side. The density of taps varied in the spanwise direction depending on where the time-averaged vortex was located from the PIV data to capture the vortex footprint more intricately.

The pressure measurement was carried out using a cylindrical 48 port Scanivalve ${ }^{\circledR}$ multiplexer, which was equipped with a Sensortechnics HCX series differential pressure transducer operating within the range -10 to +10 mbar. The Scanivalve ${ }^{\circledR}$ was placed above the working section during testing, with the pressure taps fed through the support system. Prior to testing, the transducer was calibrated using a hand held 1 bar rated Druck DPI610 pressure calibrator. Each time-averaged pressure measurement consisted of an average of 3 sets of tap readings, with 1000 readings at a sampling frequency of $1 \mathrm{kHz}$ for each pressure tap.

\subsubsection{Particle image velocimetry}

The PIV system consisted of a TSI ${ }^{\circledR} 610034$ synchronizer connected to a $120 \mathrm{~mJ}$ Nd:YAG pulsed laser. A six jet TSI ${ }^{\circledR} 9307$ oil droplet generator was used to seed the wind tunnel, with particle diameters of about $1 \mu \mathrm{m}$. A $105 \mathrm{~mm} f / 2.8 \mathrm{D}$ Nikon lens attached to a 4 Megapixel Powerview Plus CCD camera captured 1000 instantaneous image pairs for each measurement plane at a frequency of $3.75 \mathrm{~Hz}$. Images were processed in $\mathrm{TSI}^{\circledR}$ Insight $3 \mathrm{G}$ software using the Hart cross-correlation algorithm with a $32 \times 32$ interrogation area and $50 \%$ overlap. The spatial resolution varied between 0.9 and $1.5 \mathrm{~mm}$, which is less than $1 \%$ of the model diameter.

Crossflow PIV measurements (within the $y-z$ plane) were performed on each model at 5 stations along the afterbody: $x / L=0.2,0.4,0.6,0.8$ and 1.0 (Fig. 1a). Only the starboard vortex was captured, as flow symmetry is assumed. The PIV camera was positioned downstream of each model within a transparent perspex box attached to a vertical support pole. The laser was mounted on a traverse system perpendicular to the freestream. The traverse allowed the laser to be moved vertically and horizontally, while the camera box could be moved vertically along its support pole (Fig. 2). The estimated uncertainty for velocity measurements was $2 \%$ of the freestream velocity.

\subsection{Water tunnel experimental setup}

The experiments were performed in a free-surface, closed-loop water tunnel (Eidetics Model 1520) within
Fig. 2 Wind tunnel experimental setup

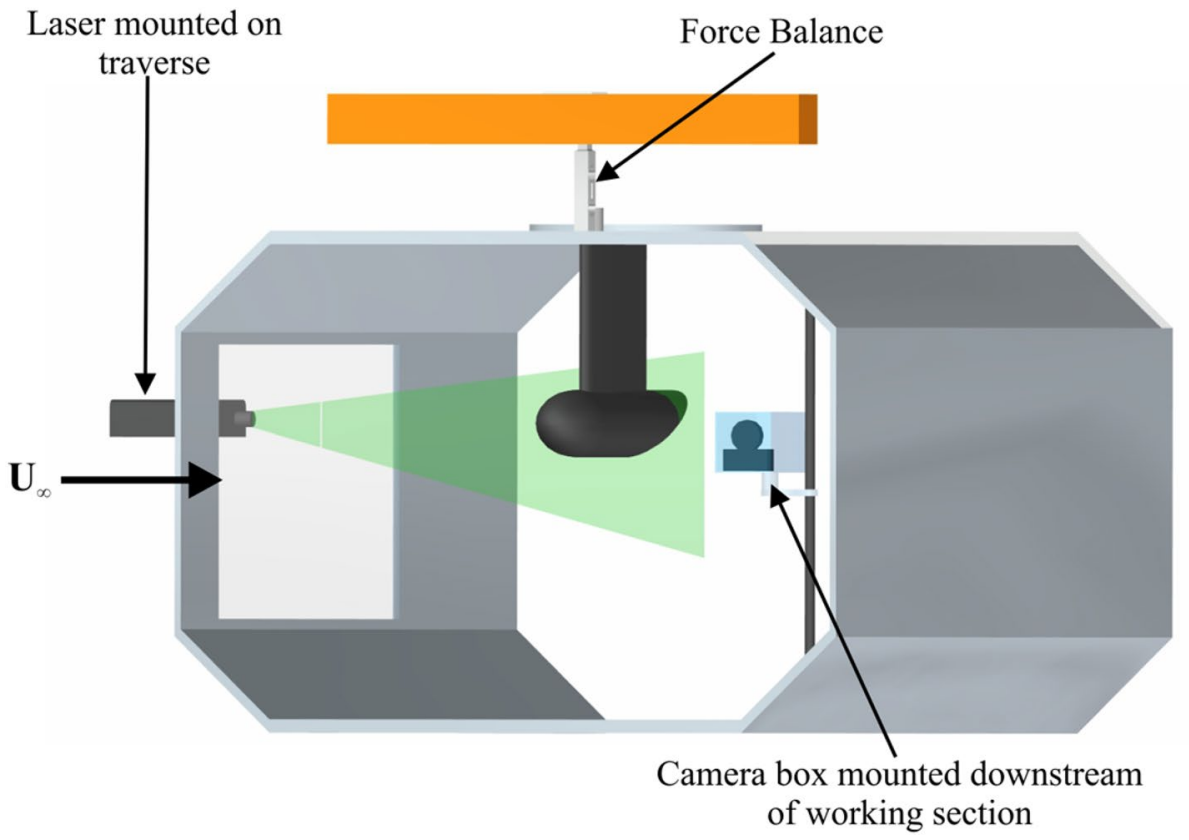


the Department of Mechanical Engineering, University of Bath. The working test area has an internal cross-section of $0.381 \times 0.508 \mathrm{~m}$, and a length of $1.530 \mathrm{~m}$. The flow velocity can increase to a maximum of $0.5 \mathrm{~m} / \mathrm{s}$, and the freestream turbulence intensity has been measured to be less than $0.5 \%$. The Reynolds number was kept constant at $R e_{D}=2 \times 10^{4}$, resulting in a freestream velocity of approximately $U_{\infty}=0.25 \mathrm{~m} / \mathrm{s}$. This is an order of magnitude lower than the wind tunnel experiments. The estimated uncertainty in setting the tunnel velocity is $2 \%$.

\subsubsection{Water tunnel model and force measurement}

The slanted base cylindrical model used was of the same geometry as that in Fig. 1a and had an upsweep angle of $28^{\circ}$, which is similar to that on a Lockheed Martin C- 130 . The cylindrical midbody had a diameter of $89 \mathrm{~mm}$ and was connected to an ellipsoidal nose with a 2:1 major to minor axis ratio. The afterbody length was $167 \mathrm{~mm}$, giving a total model length of $456 \mathrm{~mm}$. The nose cone was fabricated by a $3 \mathrm{D}$ printer out of $\mathrm{ABS}$ plastic, the midbody was made from a section of ABS pipe and the afterbody was manufactured from glass fiber reinforced plastic. The model was mounted on a support fairing with a NACA 0012 airfoil cross-section.

The drag force was measured using a Futek LSB200 'S Beam' load cell with a capacity of $0.5 \mathrm{~N}$. The signal was collected at a rate of $500 \mathrm{~Hz}$ for 45,000 samples, and was amplified using a Wheatstone bridge circuit. An end plate was mounted just below the free-surface of the water tunnel to minimise the transmission of wave oscillations to the load cell. As the drag force was smaller in the water tunnel experiments, the measurement uncertainty in the drag coefficient was higher, which was estimated to be $4 \%$.

\subsubsection{Particle image velocimetry (PIV)}

The PIV system consisted of a TSI ${ }^{\circledR} 610034$ synchronizer connected to a $200 \mathrm{~mJ}$ Nd:YAG pulsed laser. The water tunnel was seeded using hollow glass spheres with diameters of $8-12 \mu \mathrm{m}$. A $200 \mathrm{~mm} f / 2.8 \mathrm{D}$ Nikon lens attached to a 8 Megapixel Powerview Plus CCD camera captured 300 instantaneous image pairs for each measurement plane at a frequency of $3.75 \mathrm{~Hz}$. Images were processed in TSI ${ }^{\circledR}$ Insight 3G software using the Hart cross-correlation algorithm with a $32 \times 32$ interrogation area and $50 \%$ overlap, giving a spatial resolution in the range of $0.7-0.9 \mathrm{~mm}$, which is approximately $1 \%$ of the fuselage diameter. Crossflow velocity measurements were collected along the same 5 stations along the afterbody as with the wind tunnel tests: $x / L=0.2,0.4,0.6,0.8$ and 1.0. The laser was positioned from the side of the tunnel, which generated a sheet of approximately $1 \mathrm{~mm}$ thickness perpendicular to the freestream. The water tunnel setup is shown in Fig. 3. In these experiments, a relatively large area could be imaged. Therefore, both vortices of the vortex pair were measured simultaneously.
Fig. 3 Water tunnel experimental setup

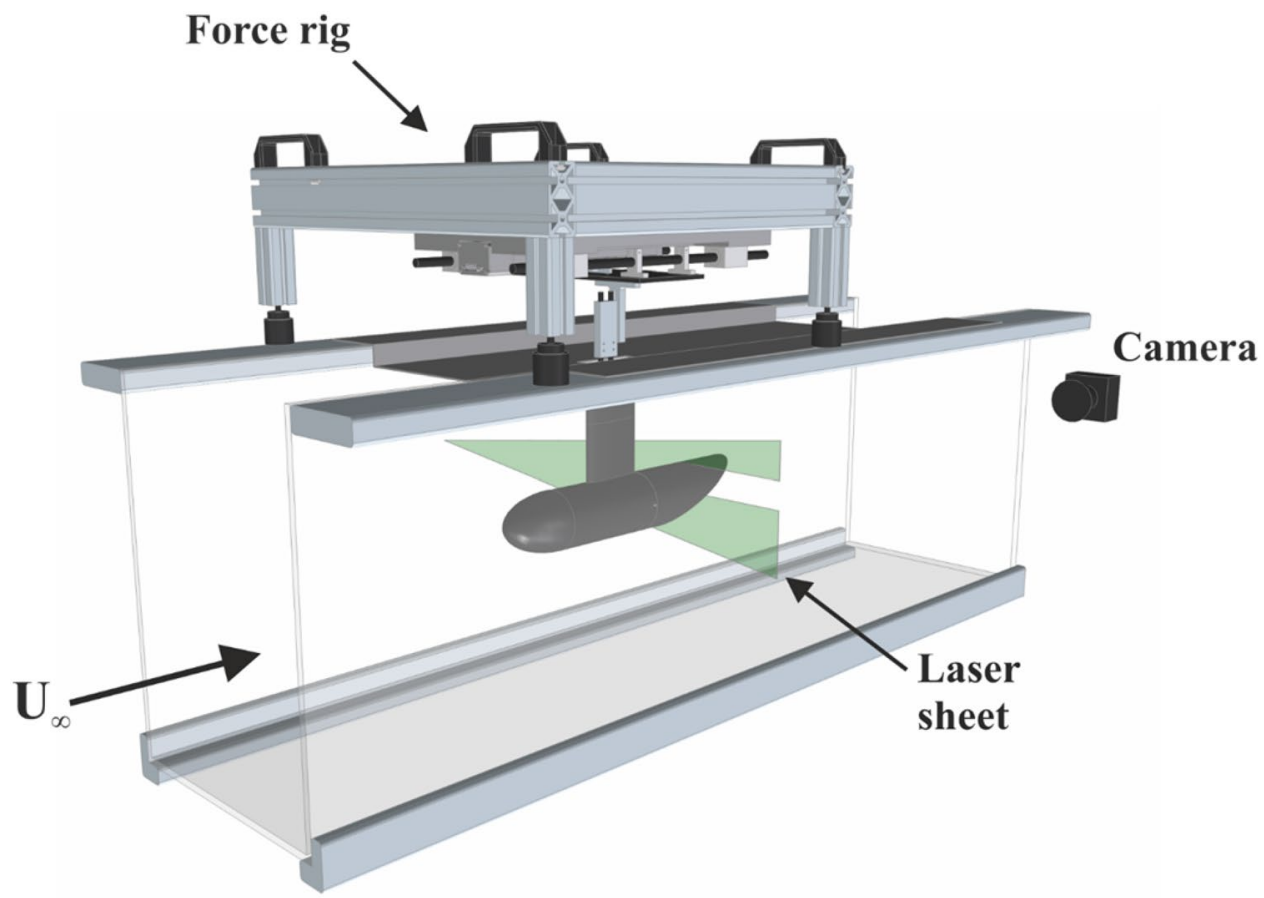




\section{Results and discussion}

\subsection{Time-averaged drag and flow}

The drag measurements are presented in Fig. 4 for all test cases alongside data from previous studies. The data collected by Stuart and Jones (1977) were extracted from the analysis by Bearman (1980). For the wind tunnel experiments, changing the upsweep angle from $\Phi=24^{\circ}$ to $32^{\circ}$ increased the drag coefficient by about $50 \%$. The wind tunnel results show good agreement with data reported in previous studies. The drag coefficient for the low Reynolds

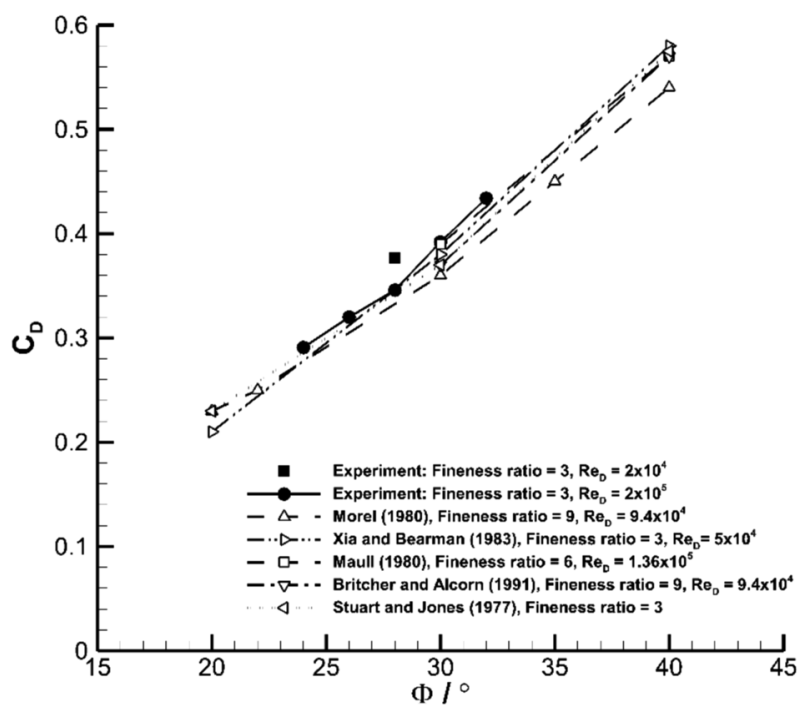

Fig. 4 Variation of drag coefficient as a function of upsweep angle and comparison with literature number water tunnel test with $\Phi=28^{\circ}$ is $9 \%$ higher compared to the wind tunnel model with the same upsweep angle. Figure 4 also shows that no significant influence of the fineness ratio could be detected. The fineness ratio is defined as the model length between the nose and the upsweep apex normalized by the diameter $D$.

The surface pressure contours along with time-averaged PIV vorticity results for the $\Phi=28^{\circ}$ wind tunnel model is shown in Fig. 5 as a perspective view, with the flow approaching diagonally from bottom right. To preserve clarity, the PIV image frame outlines are shown at each of the five measurement stations. An overall adverse pressure gradient is present along the upsweep centerline with the contours changing gradually from blue (low pressure) to red (high pressure). A low pressure vortex footprint (blue region) exists towards the edge of the ellipse which is captured well with the pressure measurements, and can be seen to exist until the $x / L=0.4$ measurement plane. At the two most upstream stations, PIV measurements show the vorticity roll-up taking place, similar to that observed over delta wings and wing tip vortices. Downstream of $x / L=0.6$, the shear layer is relatively weak compared to the rolled -up vortex, which has now started to progressively move away from the upsweep surface resulting in an increase in surface pressure. At each measurement plane, the influence of the vortex on the surface pressure can be identified by the local pressure minimum, which can be attributed to the extra pressure drag penalty due to the afterbody vortex. At the trailing-edge, the resulting fully developed vortex can be seen with an axisymmetric core region away from the surface, where the pressure has almost recovered back to the freestream value.
Fig. 5 Surface static pressure on the $\Phi=28^{\circ}$ wind tunnel model with time-averaged vorticity superimposed, $R e_{D}=2 \times 10^{5}$

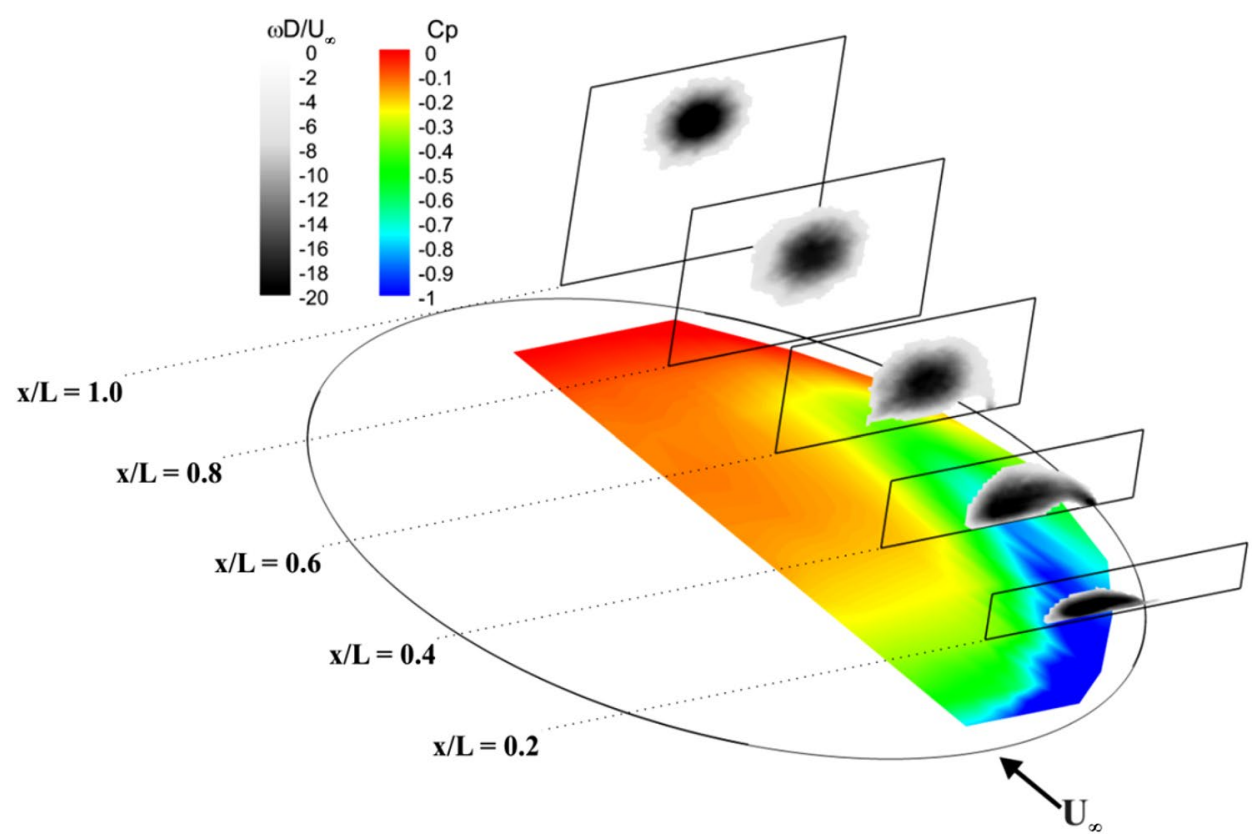


Time-averaged streamwise vorticity comparisons from the wind tunnel experiments for the two most extreme upsweep angles are presented in Fig. 6. The horizontal line represents the laser sheet intersecting the model surface. The aforementioned shear layer roll-up can be identified at $x / L=0.2$ and $x / L=0.4$ (Fig. 6a, b), but it is much weaker at $x / L=0.6$ and 0.8 . Downstream of $x / L=0.6$, the vortices become almost axisymmetric. At each measurement station, the vortex locations appear similar for the two extreme upsweep angles presented in Fig. 6. Figure 7 shows the effect of upsweep angle at $x / L=0.6$ - the first measurement plane at which the shear layer roll-up is almost complete. It can be seen that, as the upsweep angle is increased, the vorticity covers a larger area, appearing more coherent and circular. The reason for the apparent non-axisymmetric vorticity regions seen with $\Phi=24^{\circ}$ and $26^{\circ}$ cases will be examined when discussing the unsteady aspects of the flow field. The location of the vortex core does not show significant variation with the upsweep angle. At the trailing-edge, $x / L=1.0$, the vortices appear more axisymmetric for all upsweep angles as shown in Fig. 8. Again, the locations of the time-averaged vortices are similar for all upsweep angles. It can also be observed that the strength of the resulting vortex appears to increase with increasing $\Phi$.

To further assess the variation in vortex strength, the circulation for all wind tunnel test cases was calculated, and is shown in Fig. 9. The circulation was calculated using a numerical method in MATLAB ${ }^{\circledR}$ by identifying the location of the center of the vortex using the $Q$ criterion (Hunt et al. 1988; Jeong and Hussain 1995) as a first step. To calculate the $Q$ criterion, the deformation tensor, $\nabla u$, is first decomposed into symmetrical and asymmetrical components; the strain tensor, $S=0.5\left(\nabla u+\nabla u^{\mathrm{T}}\right)$ and the vorticity tensor, $\Omega=0.5\left(\nabla u-\nabla u^{\mathrm{T}}\right)$. A vortex is present in the region where $Q=0.5\left(\|\Omega\|^{2}-\|S\|^{2}\right)>0$. Vortex center is identified as the location of maximum $Q$ value. The circulation within the immediate neighborhood of this center was then calculated using an area integral of vorticity, before expanding the area outward along the grid by one spatial resolution unit and recalculating the circulation. The calculation was repeated until the change in circulation between iterations was less than $1 \%$. The $Q$ criterion was deemed an adequate vortex identification scheme for the current study since this method is capable of identifying regions of pure rotation from regions of strain (e.g., from the shear layer), as opposed to using maximum vorticity. Evaluating the circulation using this method overcomes the ambiguity associated with choosing a suitable calculation area in determining the circulation, and the effect of background noise is minimized due to the imposed $1 \%$ change criterion. Figure 9 shows that, for each upsweep angle, the circulation increases in the streamwise direction, as vorticity is continuously shed into the vortex core by means of the shear layer. The growth in circulation is more rapid at the upstream stations $x / L=0.2$ and 0.4 , suggesting the importance of the initial vortex roll-up process on the final strength of the fully developed vortex. Relatively weaker shear layer downstream contributes little to the circulation: the ratio of the circulations at $x / L=0.6$ and $x / L=1.0$ is $91 \%$ for $\Phi=24^{\circ}$ and $82 \%$ for $\Phi=32^{\circ}$. Downstream of the initial vortex roll-up (beyond $x / L=0.6$ ), an increase in upsweep angle results in an increase in the circulation, due to the formation of a stronger vortex. At the trailing-edge $(x / L=1.0)$, the difference in circulation between the two extreme upsweep angles is about $50 \%$, confirming that the resulting vortex strength is sensitive to the upsweep angle. Referring back to the drag plot in Fig. 4, the increase in drag coefficient can be attributed to this increase in vortex strength, since a stronger vortex implies a larger negative pressure acting on the surface-leading to a higher pressure drag. The non-dimensional circulation at the trailingedge, $x / L=1.0$, is plotted against the drag coefficient for the different upsweep angles in Fig. 10, revealing a linear relationship with a correlation coefficient of 0.998 . The straight line can be expressed as:

$C_{D}=0.80 \frac{\Gamma_{\mathrm{TE}}}{U_{\infty} D}+0.01$,

which reveals that the drag coefficient is nearly proportional to vortex strength at the trailing-edge.

Figure 11 presents a comparison between the time-averaged streamwise vorticity of the two different Reynolds number experiments. At the first two measurement stations $(x / L=0.2$ and 0.4$)$ where the vorticity roll-up is more pronounced, it can be observed that the vortex forms further outboard in the higher Reynolds number flow. The shear layer appears more defined at the lower Reynolds number $R e_{D}=2 \times 10^{4}$, and roll-up into the vortex is delayed. Towards the trailing-edge, the vortex is located slightly further away from the surface at the lower Reynolds number. Also, the low Reynolds number results appear to have more visible pockets of opposite vorticity close to the surface at $x / L=0.4$ and 0.6 stations (Fig. $11 \mathrm{~b}, \mathrm{c}$ ). These are bounded by the shear layer and vortex and are thought to originate from local flow separation on the model surface. The fully developed time-averaged vortex at the trailingedge, $x / L=1.0$, appears more diffused for the lower Reynolds number than the corresponding high Reynolds number case (Fig. 11e). On calculating the circulation for this measurement station, it was observed that the circulation for $R e_{D}=2 \times 10^{4}$ was $17 \%$ higher than for $R e_{D}=2 \times 10^{5}$. This suggests an overall stronger fully developed vortex present in the water tunnel which resulted in the $9 \%$ increase in its drag coefficient seen previously in Fig. 4. 
Fig. 6 Time-averaged streamwise vorticity for the $\Phi=24^{\circ}$ model (left) and $\Phi=32^{\circ}$ model (right). a $x / L=0.2$;

b $x / L=0.4 ;$ c $x / L=0.6$; d $x / L=0.8 ;$ e $x / L=1.0$. $R e_{D}=2 \times 10^{5}$ (a)

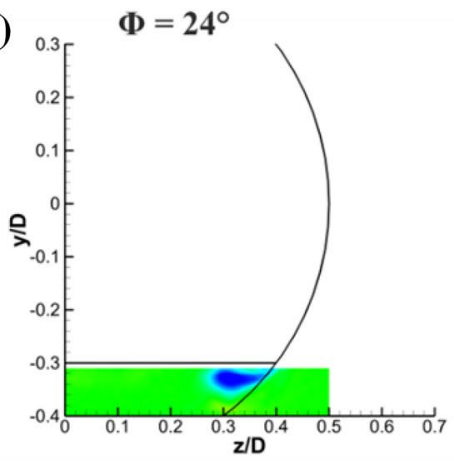

(b)

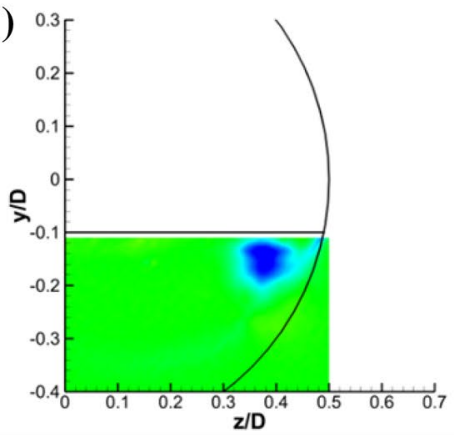

(c)

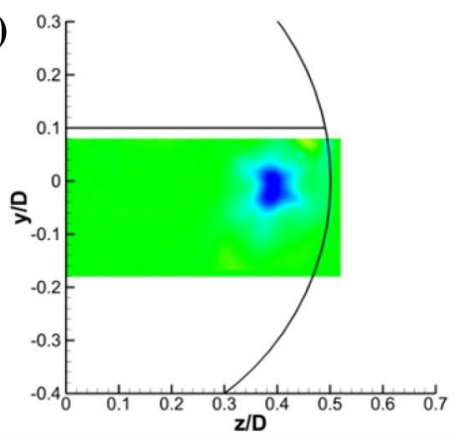

(d)

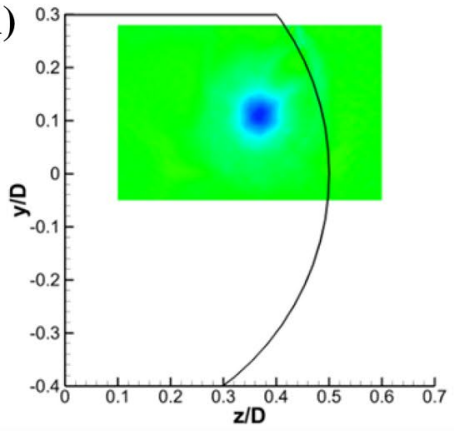

(e)

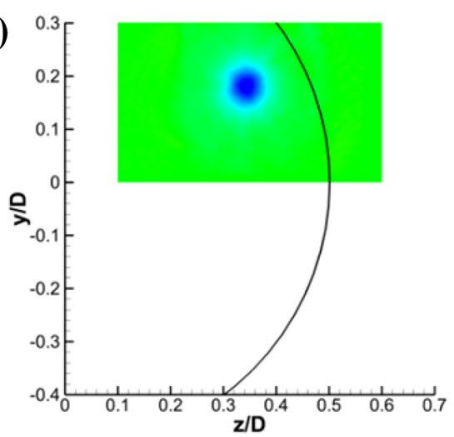

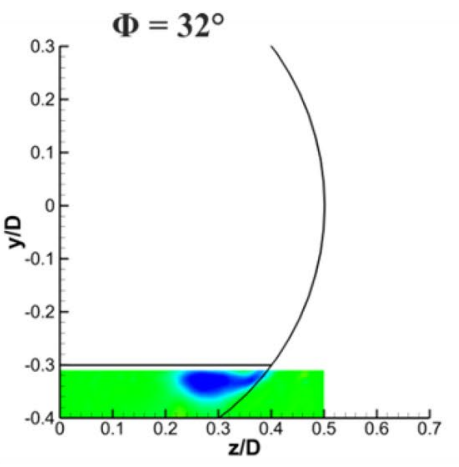
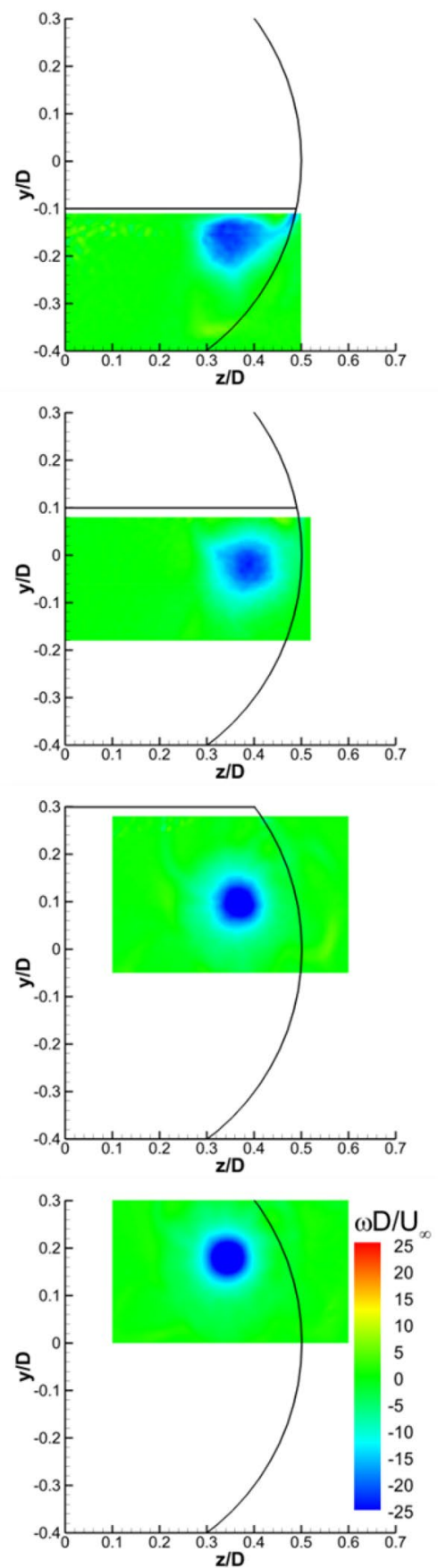

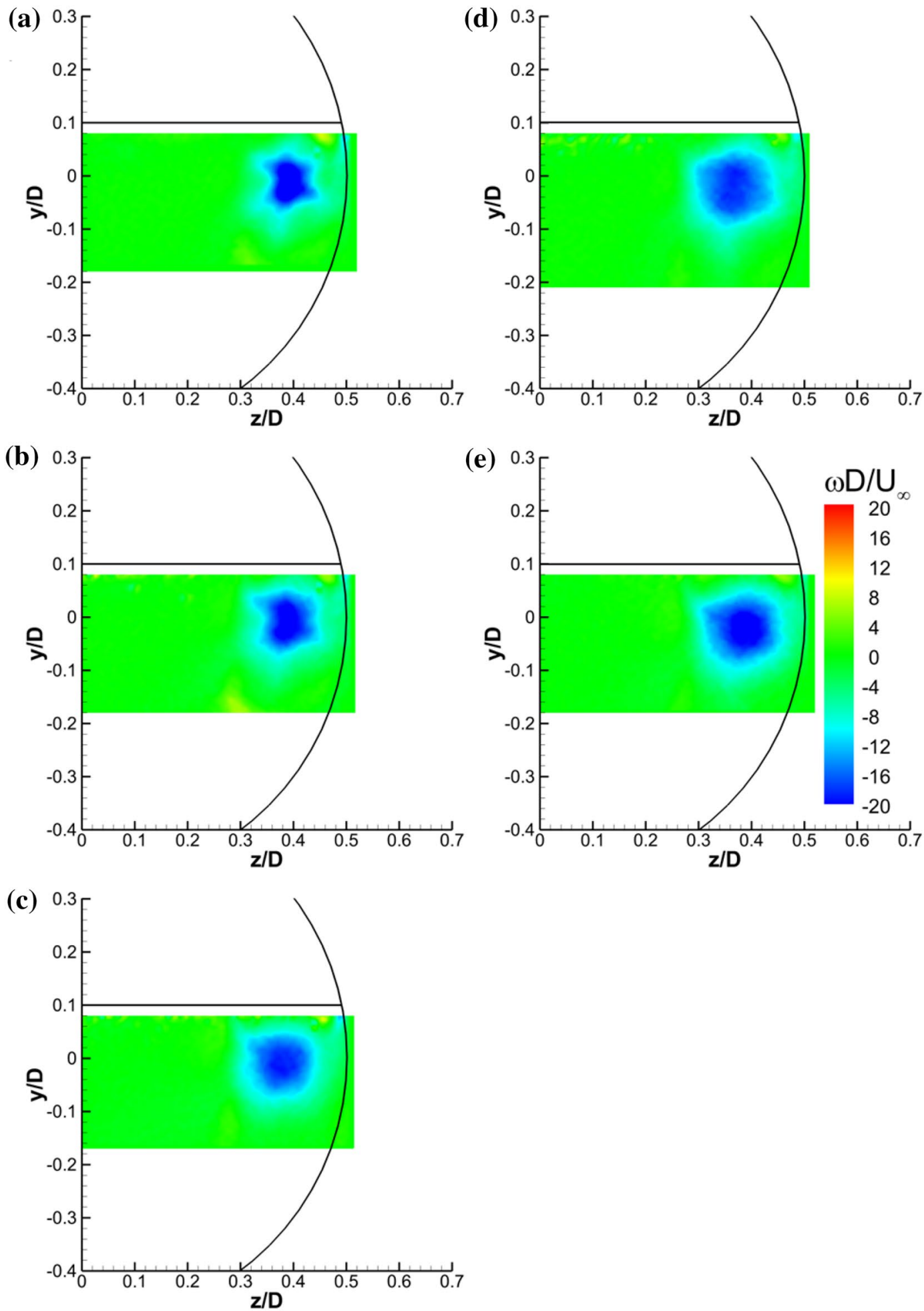

Fig. 7 Time-averaged streamwise vorticity at the $x / L=0.6$ measurement plane. a $\Phi=24^{\circ} ; \mathbf{b} \Phi=26^{\circ} ; \mathbf{c} \Phi=28^{\circ} ; \mathbf{d} \Phi=30^{\circ}$; e $\Phi=32^{\circ}$. $\operatorname{Re}_{D}=2 \times 10^{5}$ 
(a)

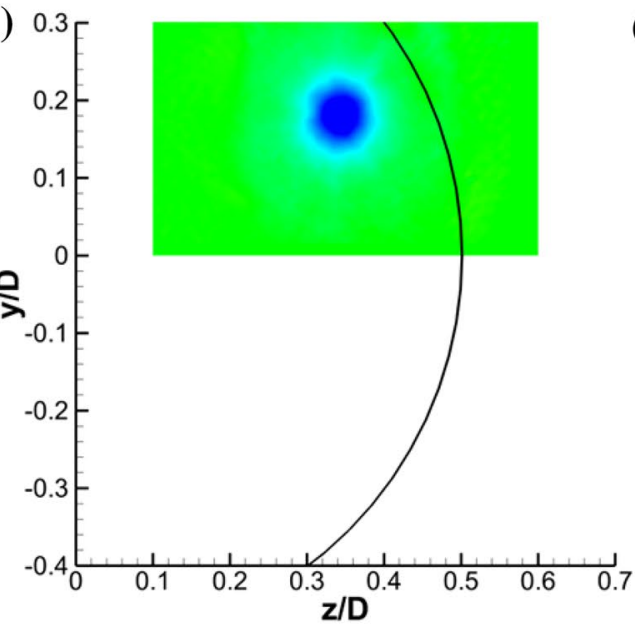

(b) 0.3

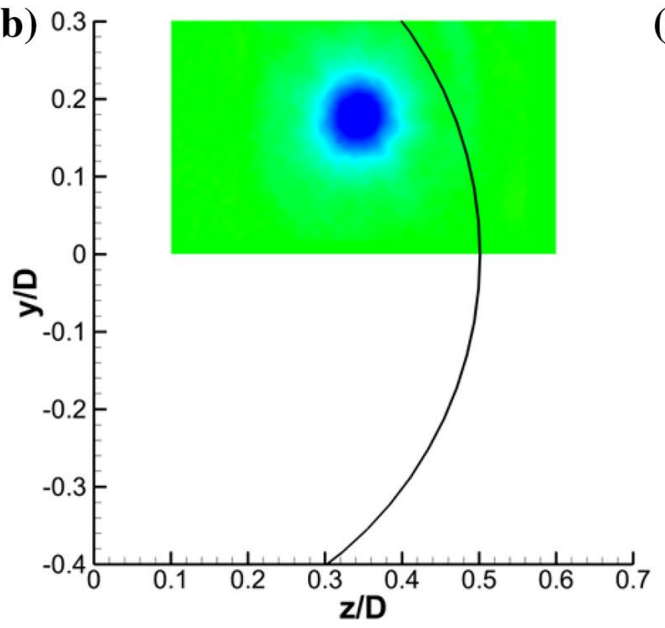

(c)

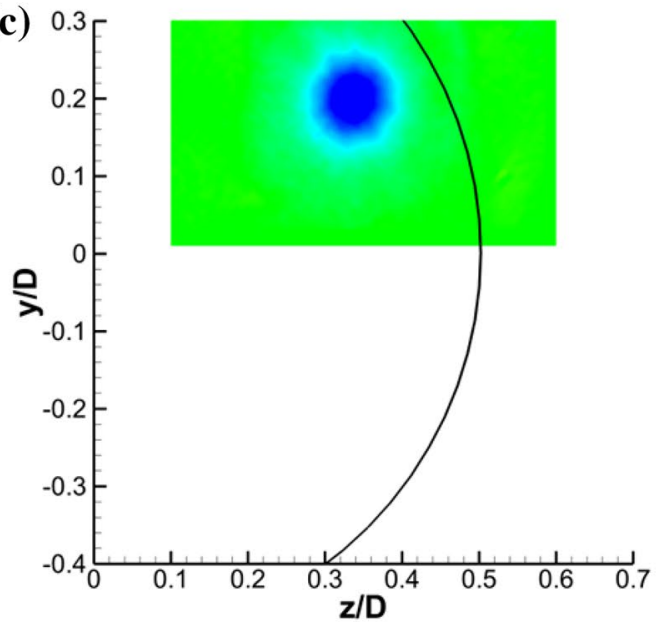

(d)

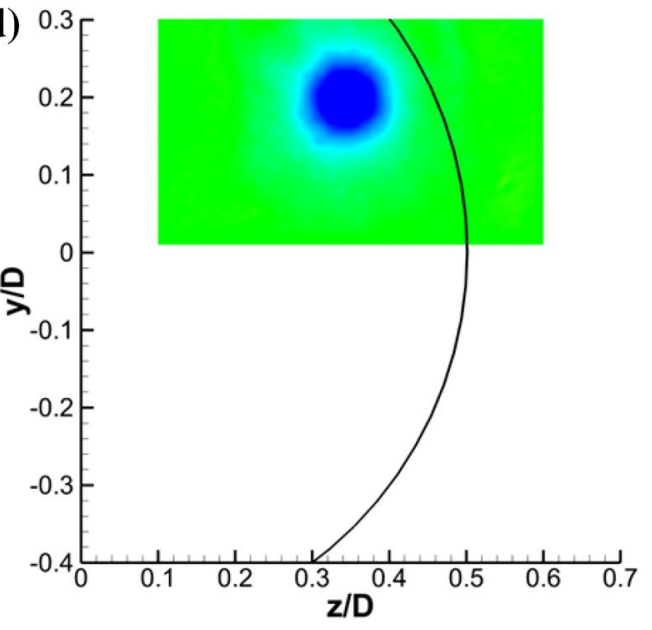

(e)

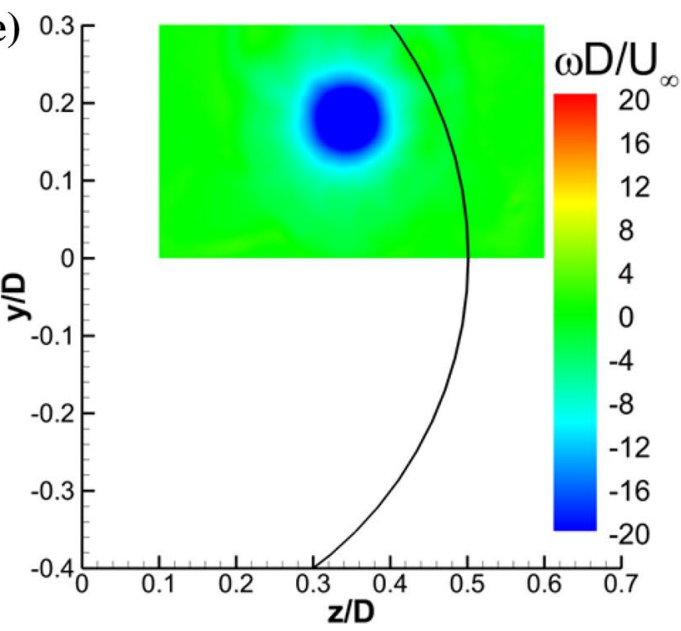

Fig. 8 Time-averaged streamwise vorticity at the $x / L=1.0$ measurement plane. a $\Phi=24^{\circ} ; \mathbf{b} \Phi=26^{\circ} ; \mathbf{c} \Phi=28^{\circ} ; \mathbf{d} \Phi=30^{\circ} ; \mathbf{e} \Phi=32^{\circ}$. $\operatorname{Re}_{D}=2 \times 10^{5}$

\subsection{Unsteady aspects}

Figure 12 presents a comparison between the timeaveraged vorticity and a corresponding instantaneous snapshot at each streamwise station for the $\Phi=32^{\circ}$ wind tunnel model. The instantaneous flow field is observed to be quite unsteady in nature. The shear layer development can be identified at the two most upstream stations, 


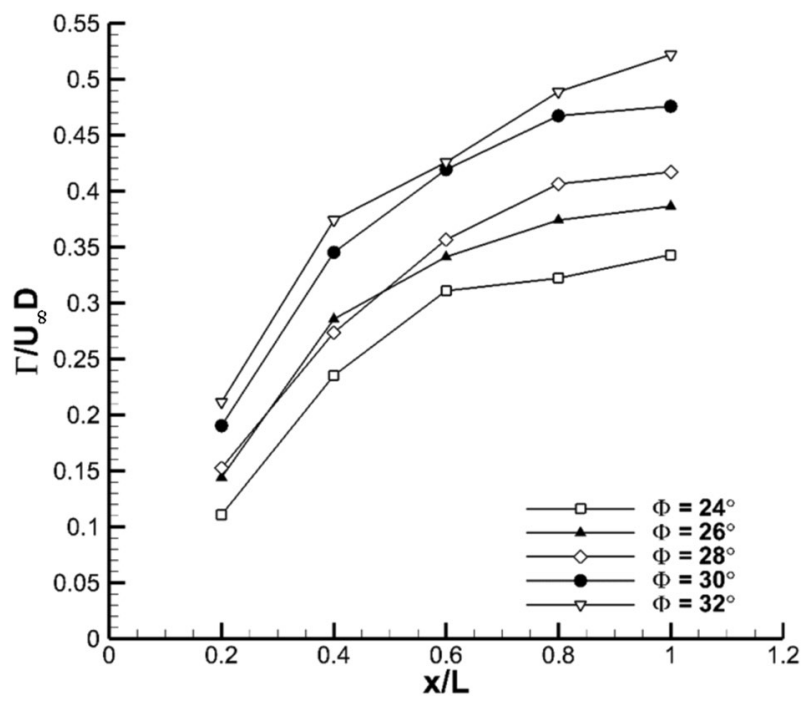

Fig. 9 Variation of non-dimensional circulation as a function of streamwise distance, $R e_{D}=2 \times 10^{5}$

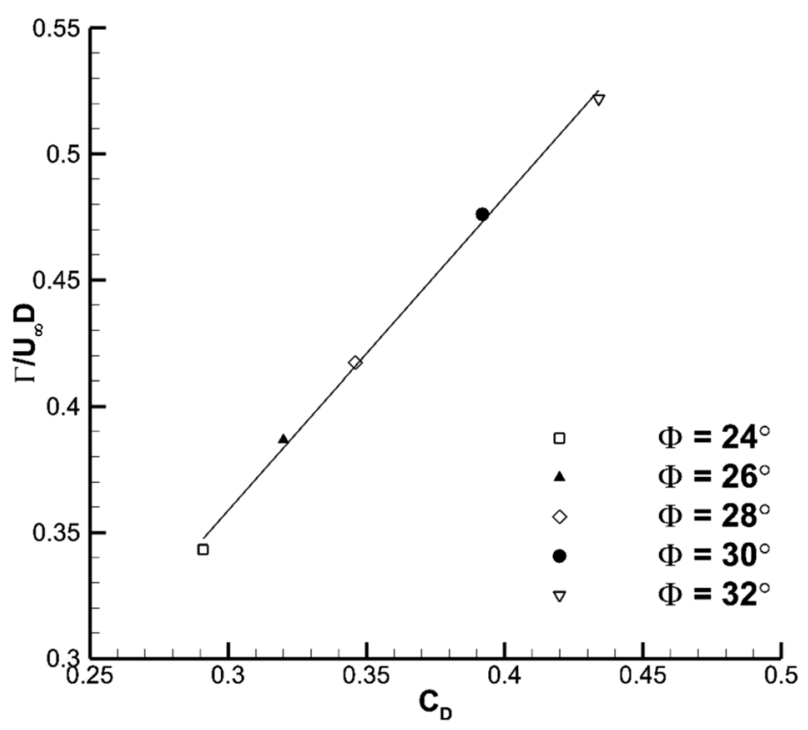

Fig. 10 Circulation at trailing-edge vs. drag coefficient, $R e_{D}=2 \times 10^{5}$

$x / L=0.2$ and 0.4. At $x / L=0.6$, the instantaneous vorticity is dispersed which seems to have caused a diffusion in the time-averaged vorticity at this location. However, at $x / L=0.8$ and 1.0 , the instantaneous vorticity is more concentrated and resembles the time-averaged vorticity to a greater extent with increased coherence. Figure 13 allows a comparison of the effect of upsweep angle on the instantaneous flow field at the trailing-edge, $x / L=1.0$. As $\Phi$ is increased, the instantaneous vorticity is less dispersed, suggesting the formation of a more coherent instantaneous vortex.
Figures 14 and 15 show the probability of the instantaneous vortex core location determined using the $Q$ criterion. These plots allow for a visualization of the vortex meandering, which cannot be deduced from the time-averaged vortex. Figure 14 provides a comparison between $\Phi=26^{\circ}$ and $32^{\circ}$ for all the PIV measurement planes, while Fig. 15 is a comparison between $x / L=0.6$ and $x / L=1.0$ measurement planes for all upsweep angles. Figure 14 suggests that the vortex meanders less towards the trailing-edge for both upsweep angles presented. This is in contrast to the observations of increasing meandering in the streamwise direction for wing tip vortices and delta wing vortices (Menke and Gursul 1997). For $\Phi=26^{\circ}$ at $x / L=0.6$ (Fig. 14c), there appears to be a presence of double peaks in the probability plot, suggesting that the vortex location appears to alternate between these two preferred locations. Referring back to Fig. 7a, b, the apparent non-circular vorticity regions seen (for $\Phi=24^{\circ}$ and $26^{\circ}$ ) in the time-averaged vorticity fields are related to these meandering characteristics. Figure $15 \mathrm{a}, \mathrm{b}$ at $x / L=0.6$ present the corresponding probability plots for the same measurement planes. It can be seen that the instantaneous vortex seems to be meandering along the vertical axis for these cases. This suggests that the deformation of the time-averaged vorticity fields in Fig. 7a, b might be due to the meandering of the vortex around two preferred locations, a behavior not observed with the larger upsweep angles beyond $\Phi=26^{\circ}$. The probability plots for $x / L=1.0$ in Fig. 15 present very similar behavior for all upsweep angles: the locations of each instantaneous vortex appear to be within small regions, indicating a decrease in vortex meandering towards the trailing-edge. The locations of these peak probabilities do not vary appreciably with increasing upsweep angle within the range under consideration.

The time-averaged vortex core radius $r_{\text {core }}$ was extracted for each wind tunnel test case based on the maximum timeaveraged velocity magnitude within the crossflow plane. A radial distance was first calculated for each PIV grid point away from the vortex center using MATLAB ${ }^{\circledR}$, and the velocity magnitudes were averaged at each radius resulting in an azimuthal average. The results are presented in Fig. 16. There is an initial rapid increase in core radius up to $x / L=0.6$ for all upsweep angles as the shear layer keeps feeding vorticity into the vortex core. Downstream of the initial vortex roll-up (beyond $x / L=0.6$ ) even though the circulation continues to increase (referring back to Fig. 9), a reduction in core radius is observed for all upsweep angles towards the trailing-edge, indicating a tightening of the vortex core. This reduction of core radius in the streamwise direction has not been observed in other external vortex flows such as wing tip vortices and delta wing vortices.

The vortex meandering amplitude $a_{m}$ is an integral measure of the deviation of each instantaneous vortex 
Fig. 11 Effect of Reynolds number for the $\Phi=28^{\circ}$ model. a $x / L=0.2 ; \mathbf{b} x / L=0.4$; c $x / L=0.6 ; \mathbf{d} x / L=0.8 ; \mathbf{e}$ $x / L=1.0$
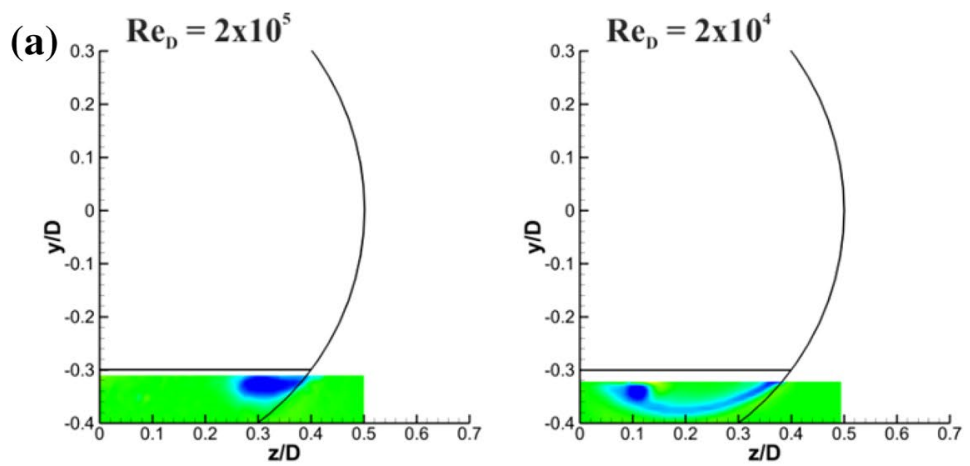

(b)
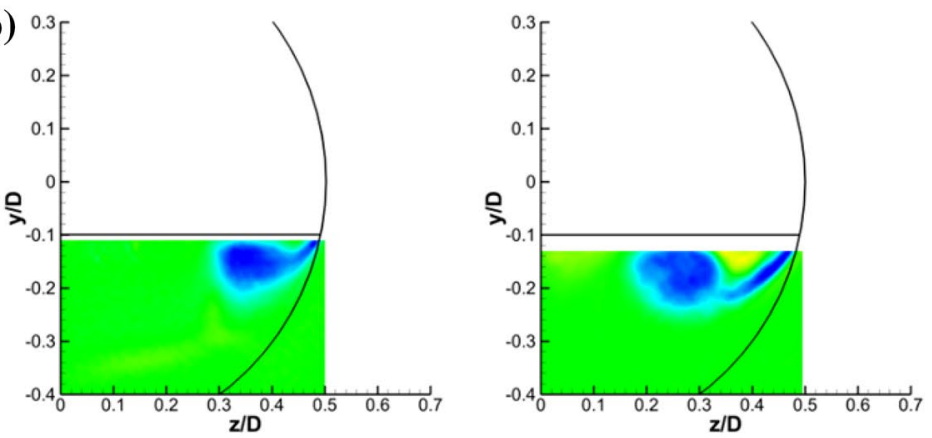

(c)
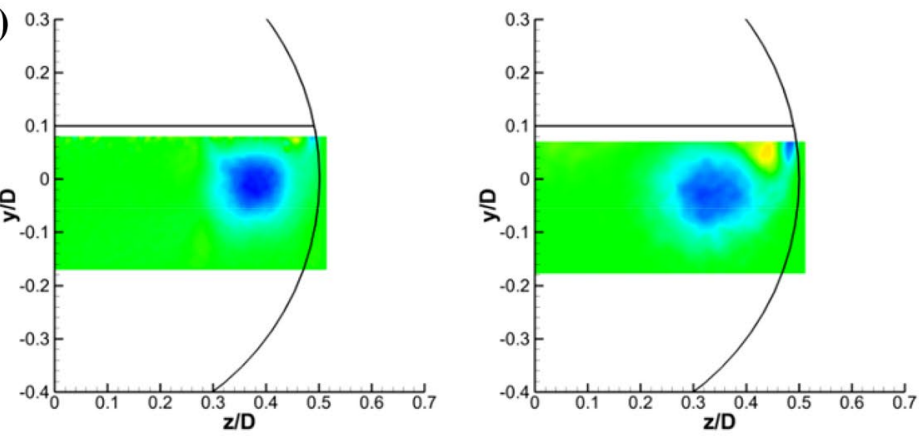

(d)
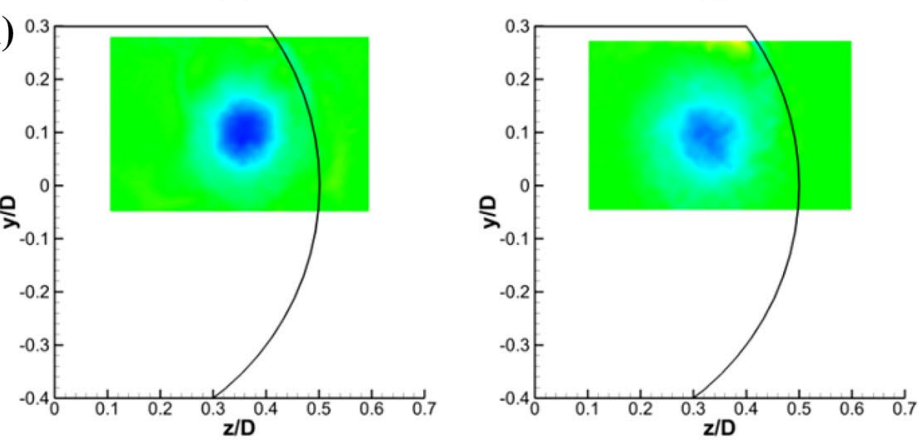

(e)

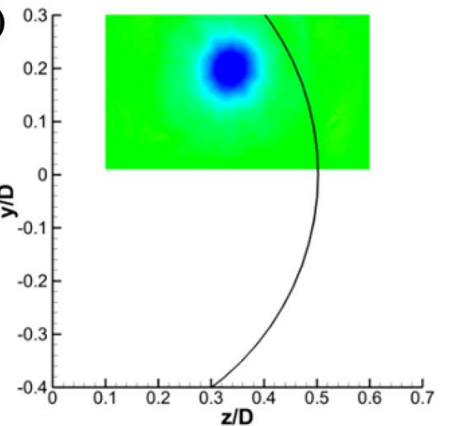


Fig. 12 Comparison of timeaveraged (left) and instantaneous (right) vorticity for the $\Phi=32^{\circ}$ model. a $x / L=0.2$; b $x / L=0.4 ;$ c $x / L=0.6$; d $x / L=0.8 ;$ e $x / L=1.0$. $R e_{D}=2 \times 10^{5}$ (a)

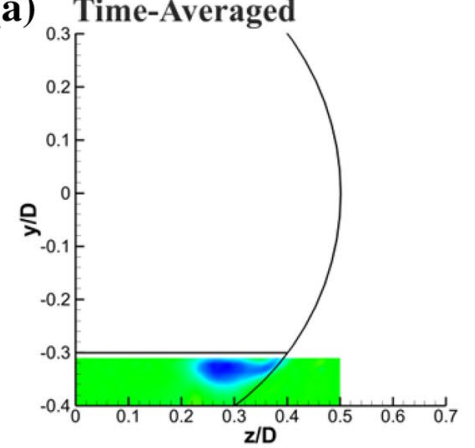

(b)

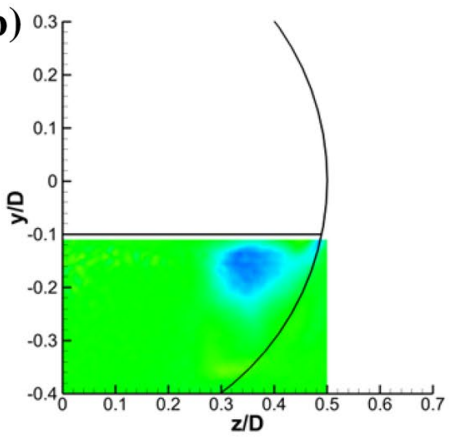

(c)

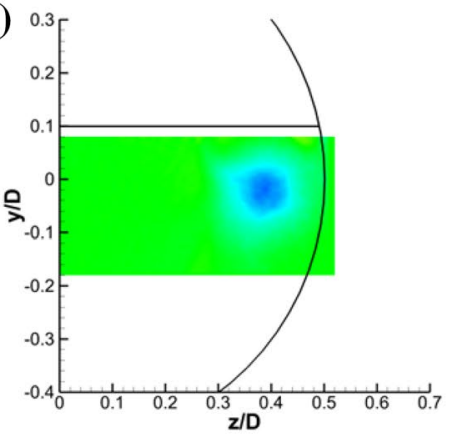

(d)

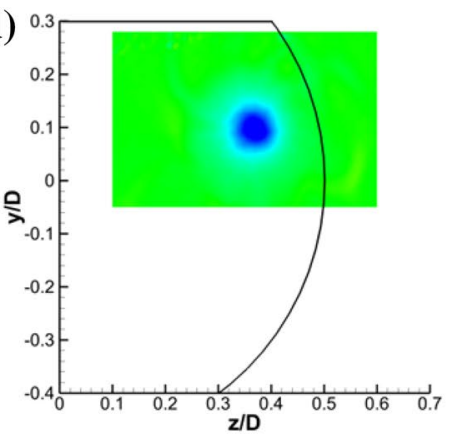

(e)

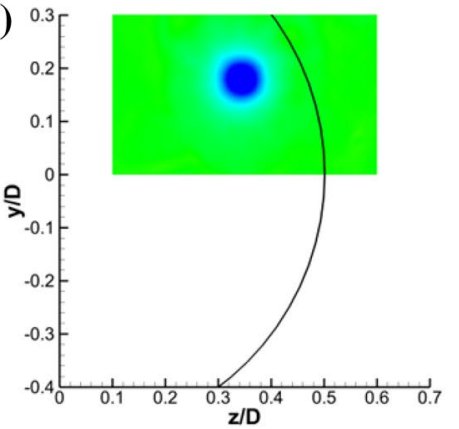

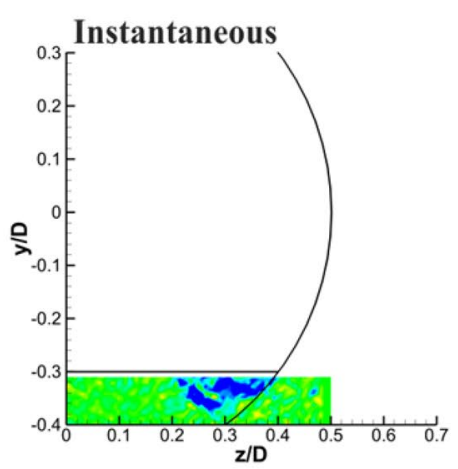
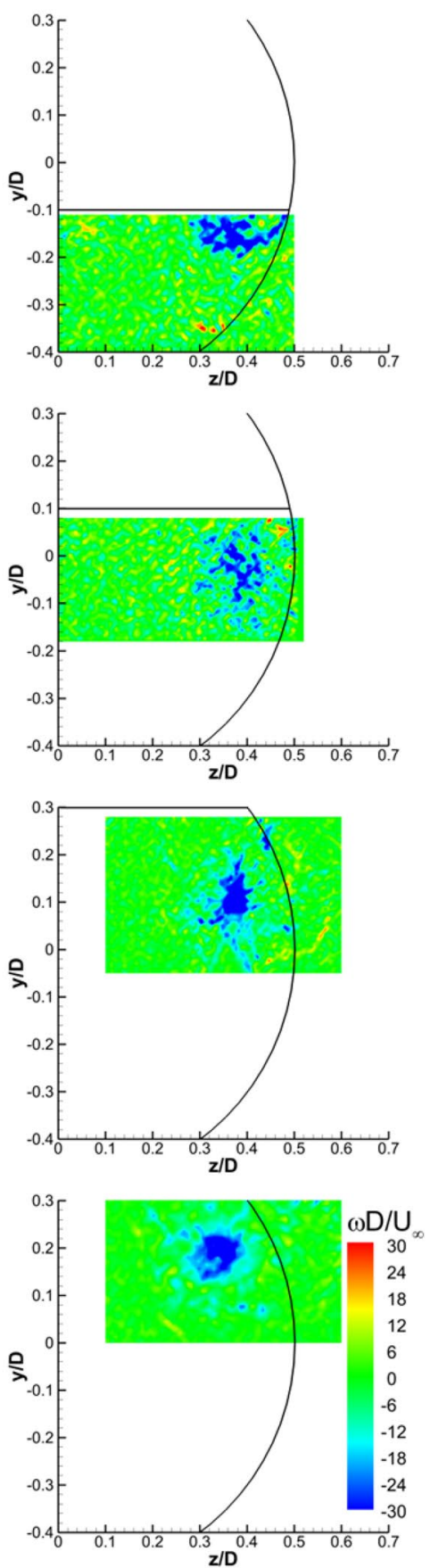


\section{Time-Averaged}

(a)

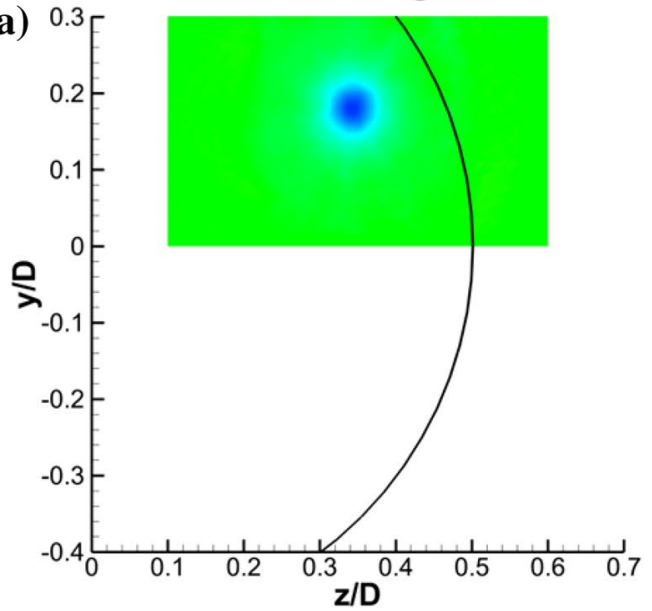

(b)

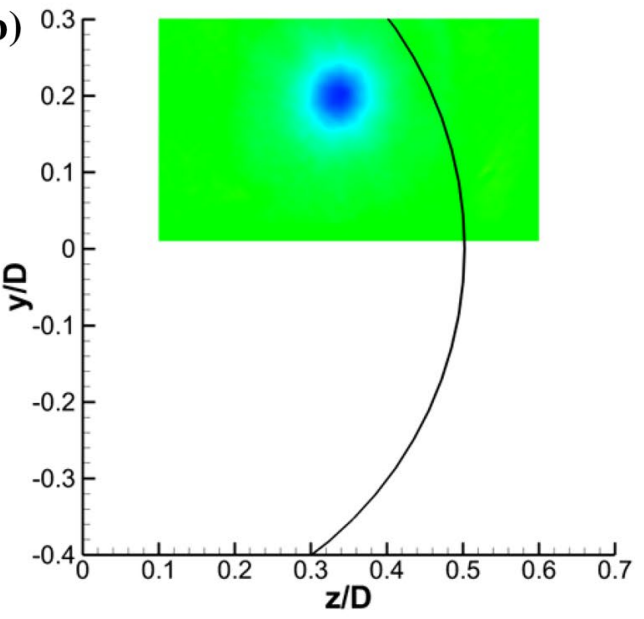

(c)

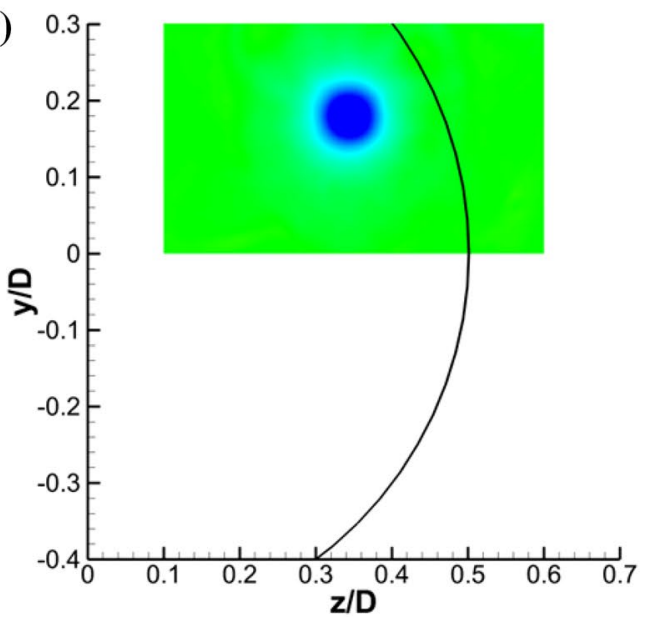

Instantaneous
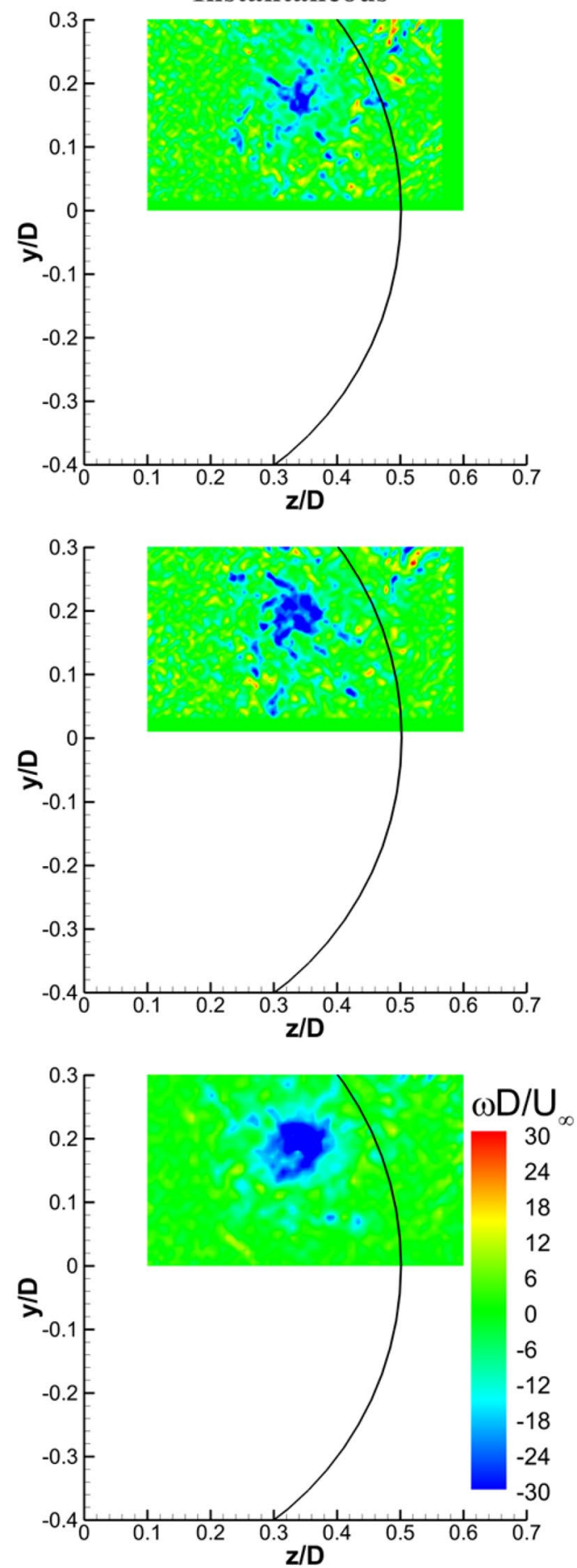

Fig. 13 Comparison of time-averaged (left) vs. instantaneous (right) vorticity at $x / L=1.0 . \mathbf{a} \Phi=24^{\circ} ; \mathbf{b} \Phi=28^{\circ} ; \mathbf{c} \Phi=32^{\circ} . R e_{D}=2 \times 10^{5}$

core location with respect to the time-averaged core location (identified using the $Q$ criterion). It is defined by:

$a_{m}=\left(\frac{1}{N} \sum_{i=1}^{N}\left(z_{i}-z_{c}\right)^{2}+\left(y_{i}-y_{c}\right)^{2}\right)^{\frac{1}{2}}$.
Figure 17a shows the variation of $a_{m}$ with streamwise distance for all upsweep angles, while Fig. 17b is a plot of the ratio $a_{m} / r_{\text {core }}$ in the streamwise direction. In terms of the variation of $a_{m}$ in Fig. 17a, the two smallest upsweep angles $\Phi=24^{\circ}$ and $26^{\circ}$ exhibit similar behavior: a gradual 
Fig. 14 Probability of instantaneous vortex location for $\Phi=26^{\circ}$ (left) and $\Phi=32^{\circ}($ right $)$. a $x / L=0.2$;

b $x / L=0.4 ;$ c $x / L=0.6$; d $x / L=0.8 ; \mathbf{e} x / L=1.0$. $R e_{D}=2 \times 10^{5}$
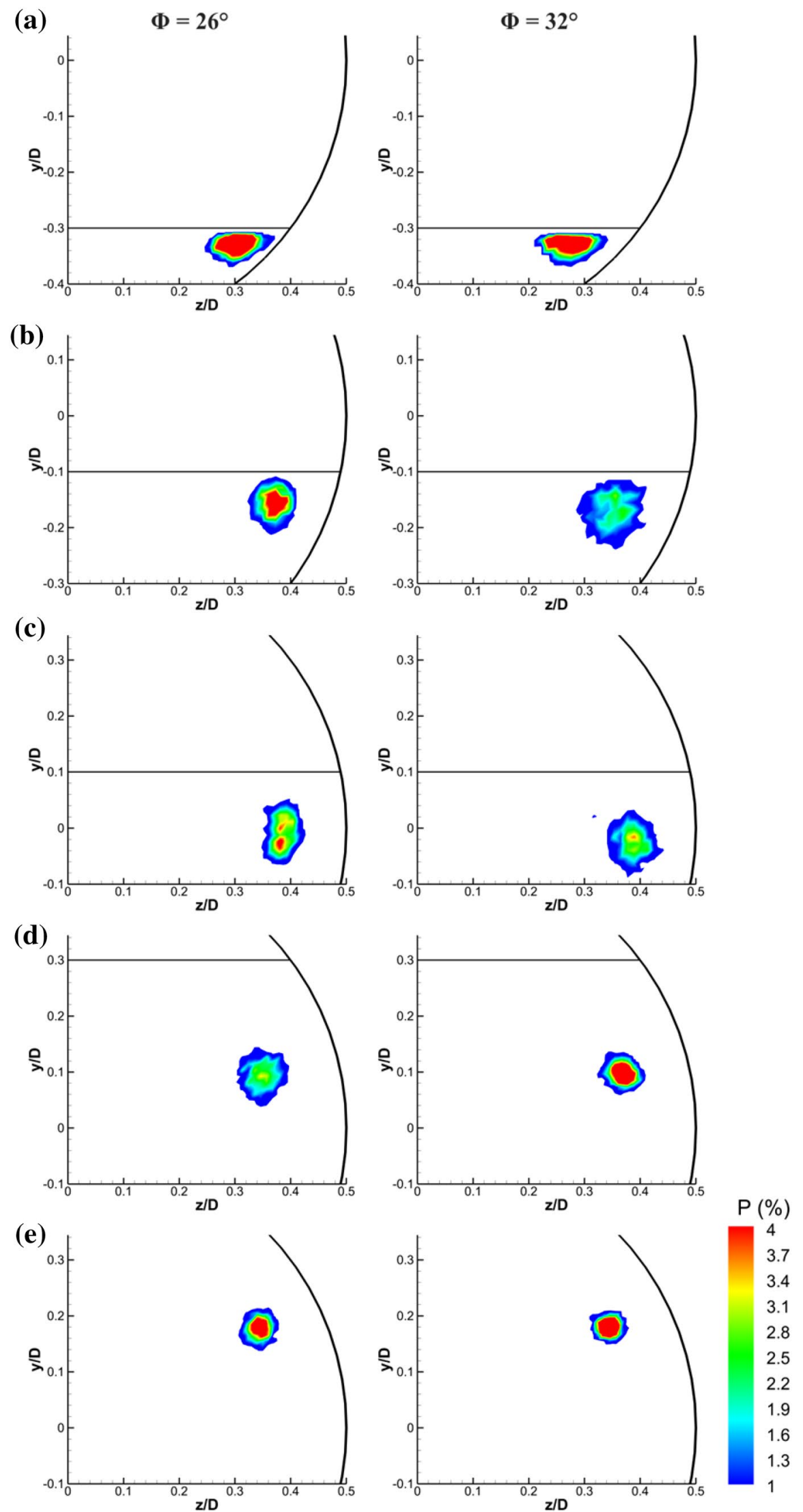
Fig. 15 Probability of instantaneous vortex location at $x / L=0.6($ left $)$ and $x / L=1.0$ (right). a $\Phi=24^{\circ} ; \mathbf{b} \Phi=26^{\circ}$; c $\Phi=28^{\circ} ; \mathbf{d} \Phi=30^{\circ} ; \mathbf{e}$ $\Phi=32^{\circ} . R e_{D}=2 \times 10^{5}$ $\mathrm{x} / \mathrm{L}=0.6$

(a)

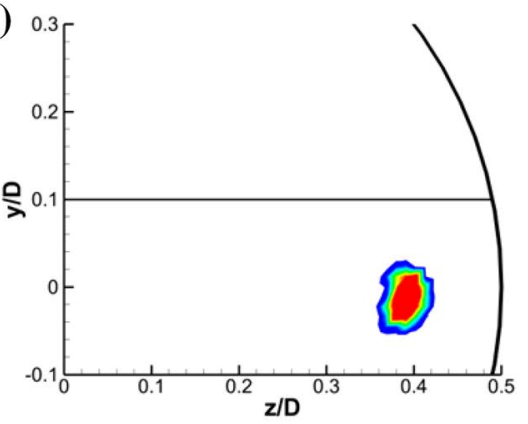

(b)

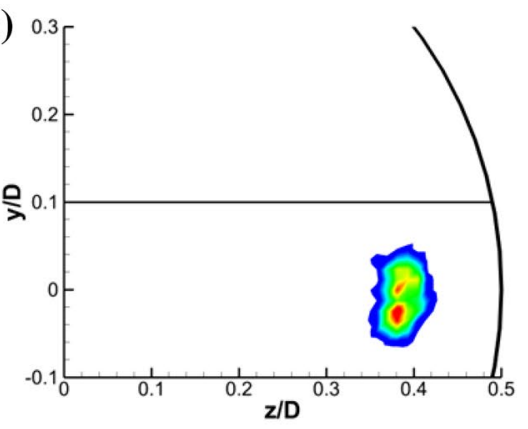

(c)

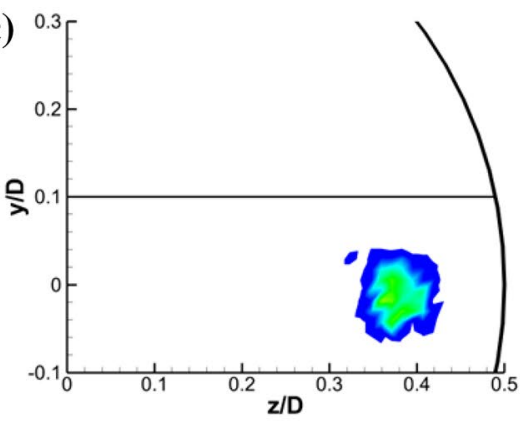

(d)

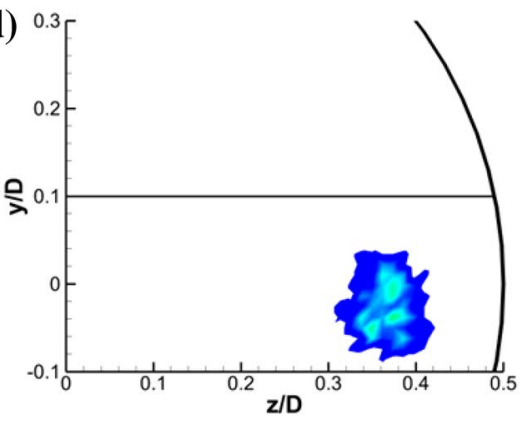

(e)

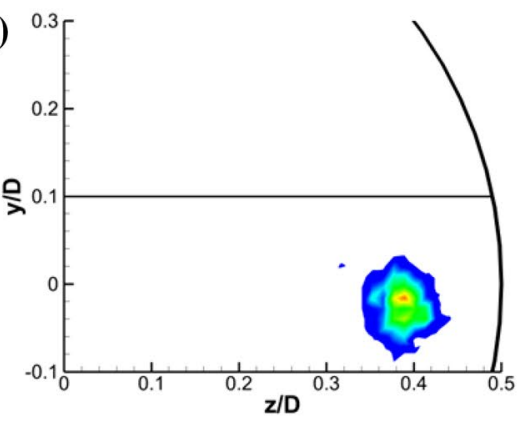

$\mathbf{x} / \mathrm{L}=\mathbf{1 . 0}$
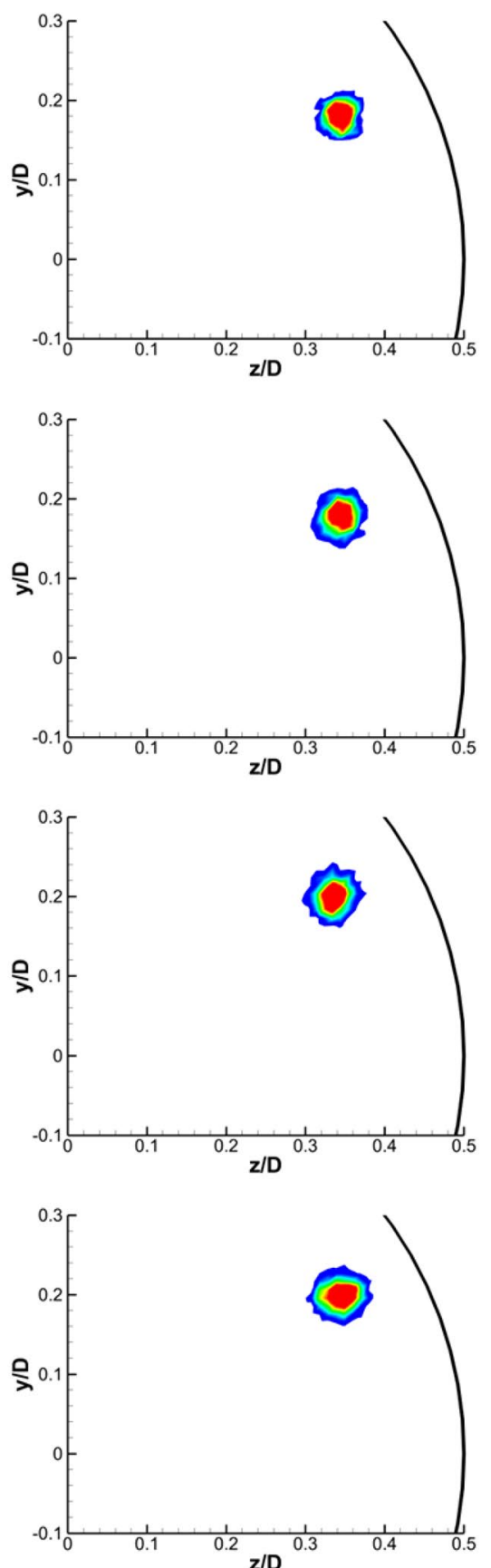

$\mathrm{P}(\%)$

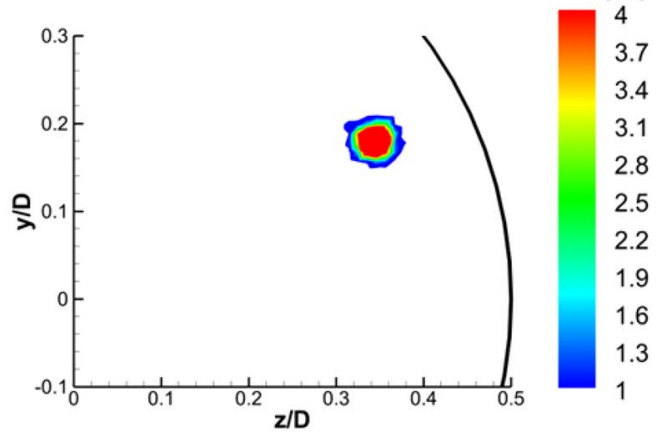




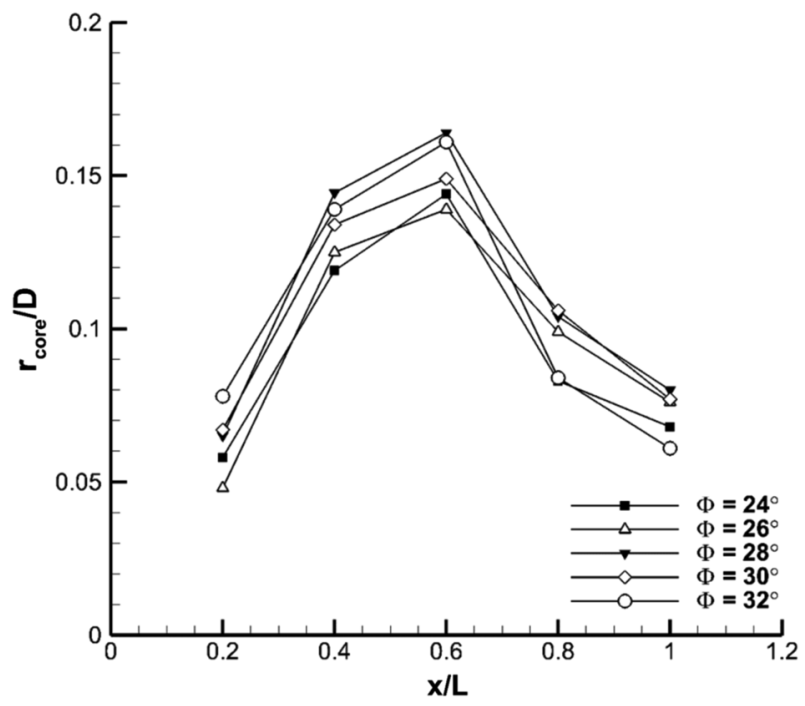

Fig. 16 Variation of time-averaged vortex core radius as a function of streamwise distance, $\operatorname{Re}_{D}=2 \times 10^{5}$

increase in $a_{m}$ is observed until $x / L=0.8$, followed by a reduction towards the trailing-edge at $x / L=1.0$. Probability plots shown in Fig. 14 for $\Phi=26^{\circ}$ are consistent with this variation. Compared to the larger upsweep angles at stations preceding $x / L=0.6$, the $\Phi=24^{\circ}$ and $26^{\circ}$ cases have smaller $a_{m}$ values, which is also consistent with Fig. 15 . The streamwise station at which the peak meandering amplitude is observed moves upstream with increasing upsweep angle. For example, for $\Phi=32^{\circ}$, the peak amplitude is found at $x / L=0.4$, which is consistent with the probability plots in Fig. 14. A general decreasing trend in $a_{m}$ is observed towards the trailing-edge for all upsweep angles. There is a close relationship between the variations of vortex core radius and amplitude of meandering in the streamwise direction. This suggests that the afterbody vortex at the trailing-edge $x / L=1.0$ exhibits reduced meandering after the vortex roll-up is complete. This reduction of meandering in the streamwise direction has not been observed with external aerodynamic vortex flows previously.

The ratio $a_{m} / r_{\text {core }}$ presented in Fig. $17 \mathrm{~b}$ provides a means of comparing the effect of meandering on the time-averaged vortex core radius. Although there is a decreasing trend between $x / L=0.2$ and 0.6 , and an increasing trend between $x / L=0.6$ and 0.8 , the ratio $a_{m} / r_{\text {core }}$ is approximately $0.3-0.4$ towards the trailing-edge $x / L=1.0$. This value is similar to those reported for wing tip vortices previously (Margaris et al. 2008; Devenport et al. 1996).

\subsection{Proper orthogonal decomposition (POD)}

The Proper Orthogonal Decomposition (POD) provides a mathematical means of extracting the major salient (most
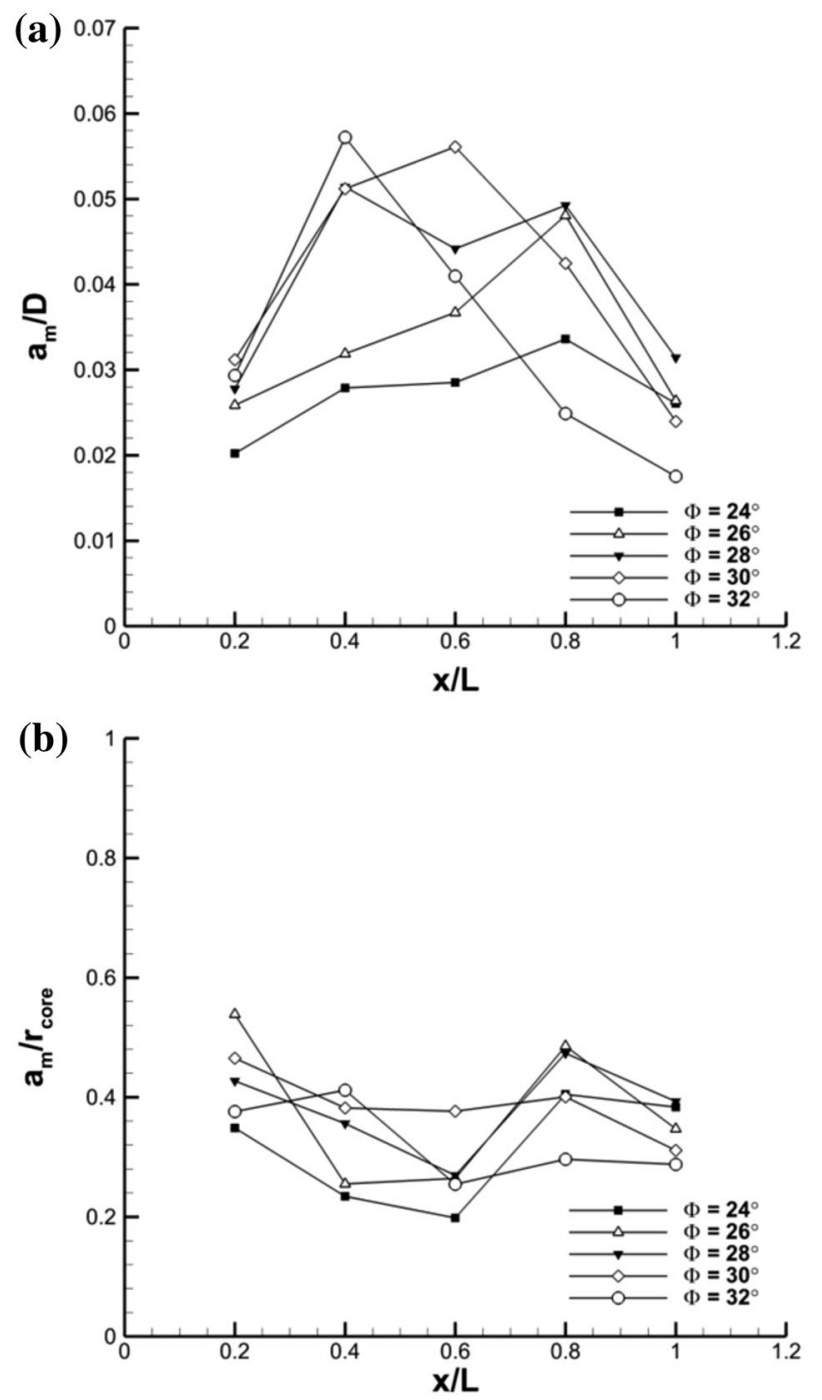

Fig. 17 Variation vortex meandering amplitude, normalized by: a diameter; $\mathbf{b}$ vortex core radius, as a function of streamwise distance, $R e_{D}=2 \times 10^{5}$

energetic) coherent flow structures that may otherwise be hidden within the instantaneous snapshots. Detailed explanations of the mathematics behind the technique can be found in Berkooz et al. (1993). The technique has been applied extensively in turbulent flows (Adrian et al. 2000) and vortex-dominated flows (Graftieaux et al. 2001; Wang and Gursul 2012) previously. In this paper, the analysis was performed using a MATLAB ${ }^{\circledR}$ code (Chen et al. 2012, 2013) which adopts the method of snapshots. The calculation was performed on the fluctuating component of the velocity field. For all of the cases considered, it was seen that only the first two modes were significant. Energy of other modes beyond the first two modes were much lower. In the discussion that follows, only the first two dominant modes are presented. 
Fig. 18 POD mode 1 (left) and mode 2 (right), for $\Phi=24^{\circ}$.

a $x / L=0.2 ; \mathbf{b} x / L=0.4$;

c $x / L=0.6 ; \mathbf{d} x / L=0.8 ; \mathbf{e}$

$x / L=1.0 . \operatorname{Re}_{D}=2 \times 10^{5}$
$\Phi=24^{\circ}$

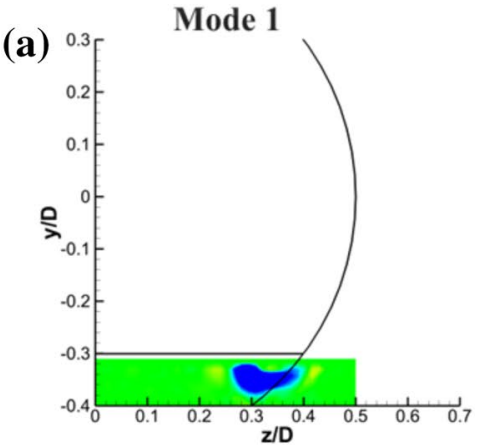

(b)

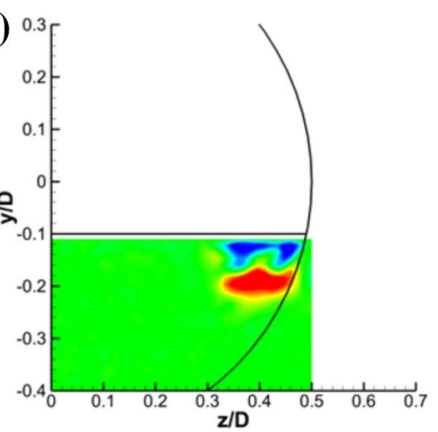

(c)

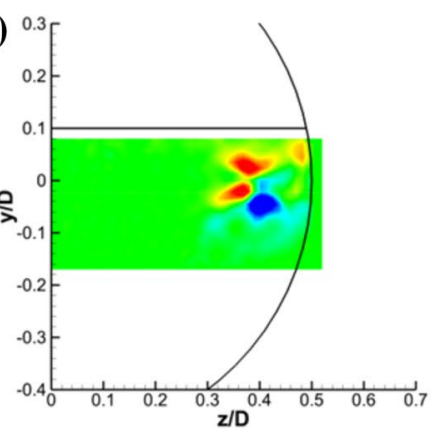

(d)

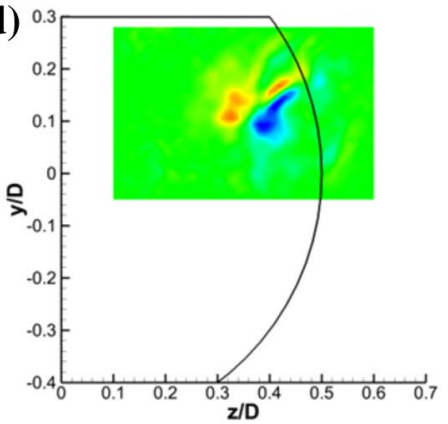

(e)

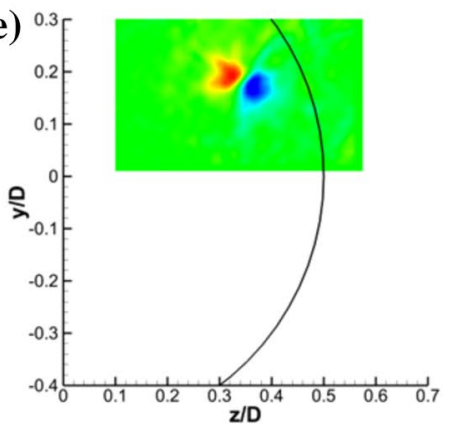

Mode 2
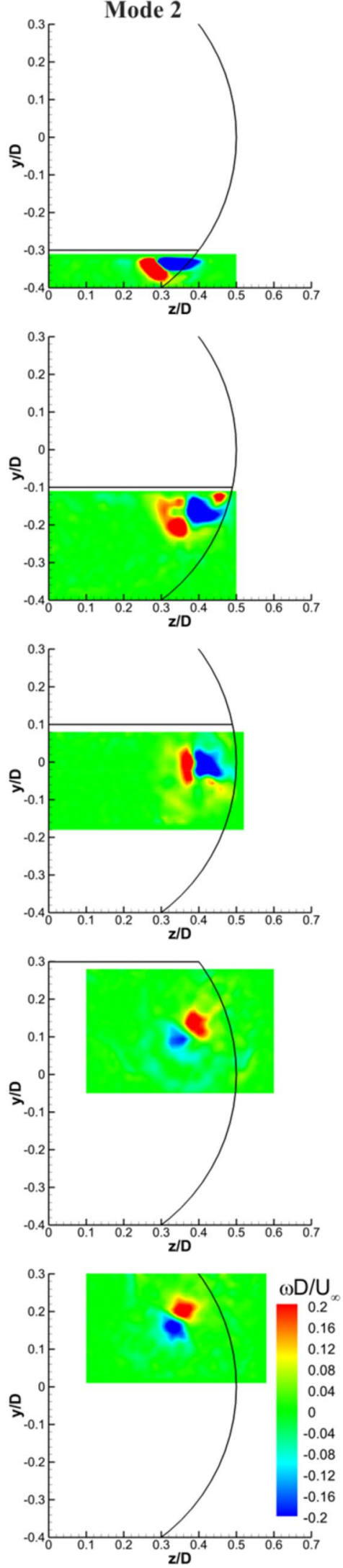
Fig. 19 POD mode 1 (left) and mode 2 (right), for $\Phi=32^{\circ}$.

a $x / L=0.2 ; \mathbf{b} x / L=0.4$;

c $x / L=0.6 ; \mathbf{d} x / L=0.8 ; \mathbf{e}$

$x / L=1.0 . R e_{D}=2 \times 10^{5}$

$\Phi=32^{\circ}$

(a)

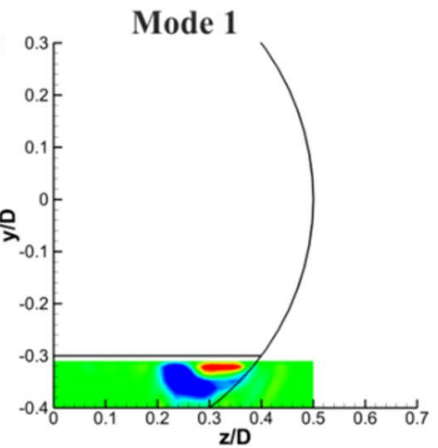

(b)

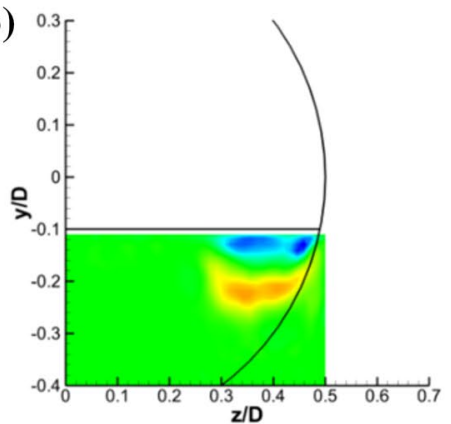

(c)

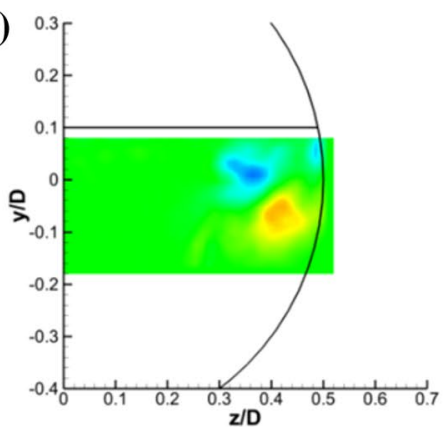

(d)

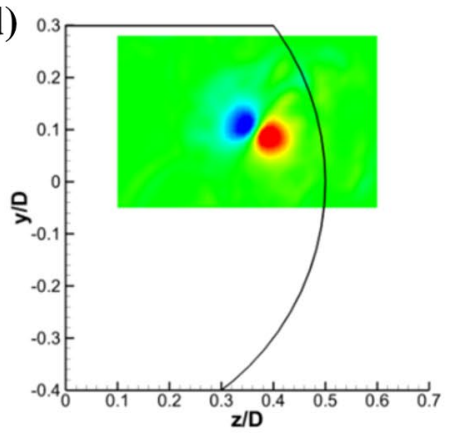

(e)

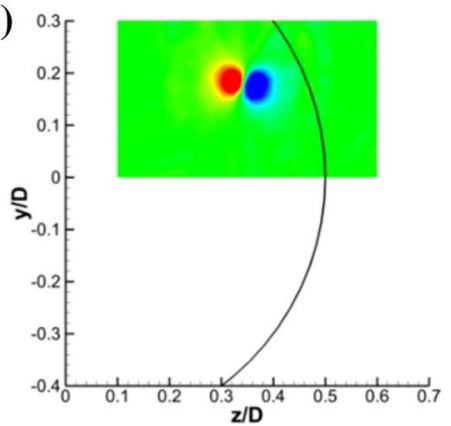

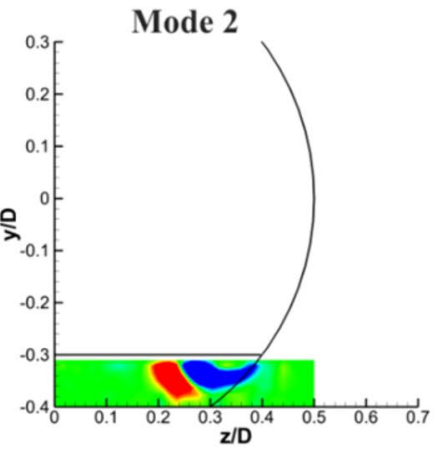
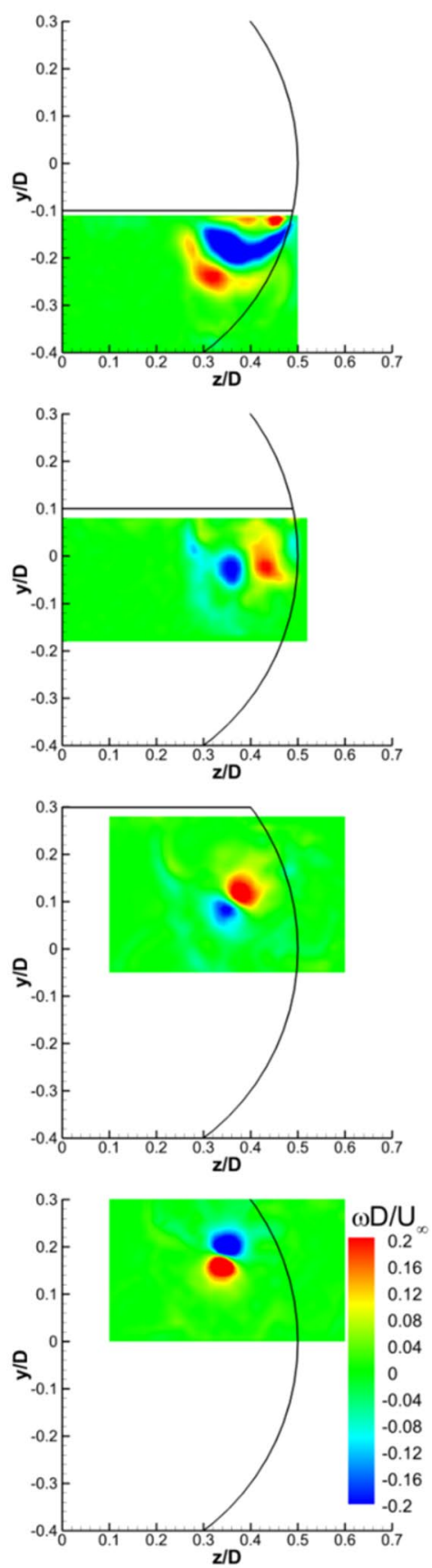
Fig. 20 POD mode 1 (left) and mode 2 (right) at $x / L=1.0$. a $\Phi=24^{\circ} ; \mathbf{b} \Phi=26^{\circ}$; c $\Phi=28^{\circ} ; \mathbf{d} \Phi=30^{\circ} ; \mathbf{e}$ $\Phi=32^{\circ} . R e_{D}=2 \times 10^{5}$ $\mathrm{x} / \mathrm{L}=\mathbf{1 . 0}$
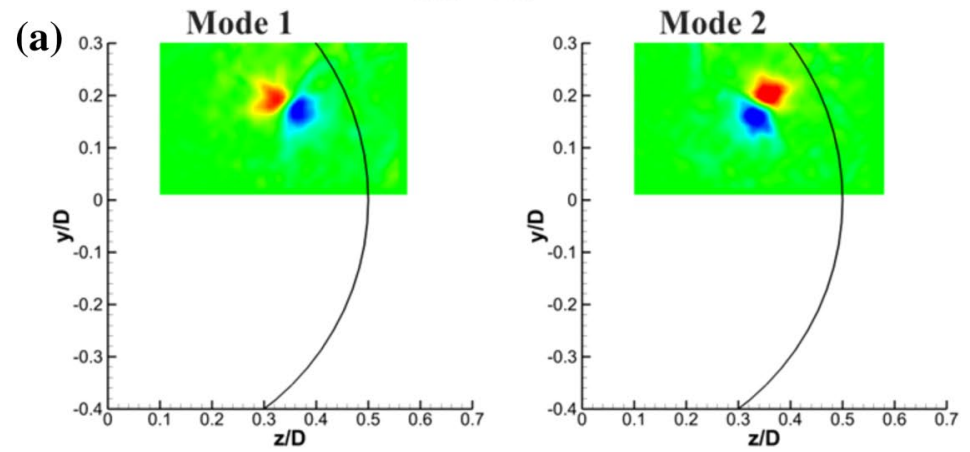

(b)
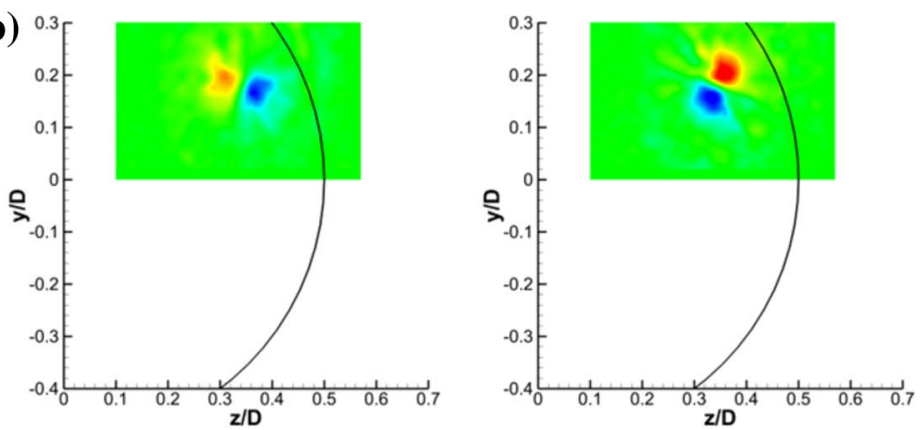

(c)
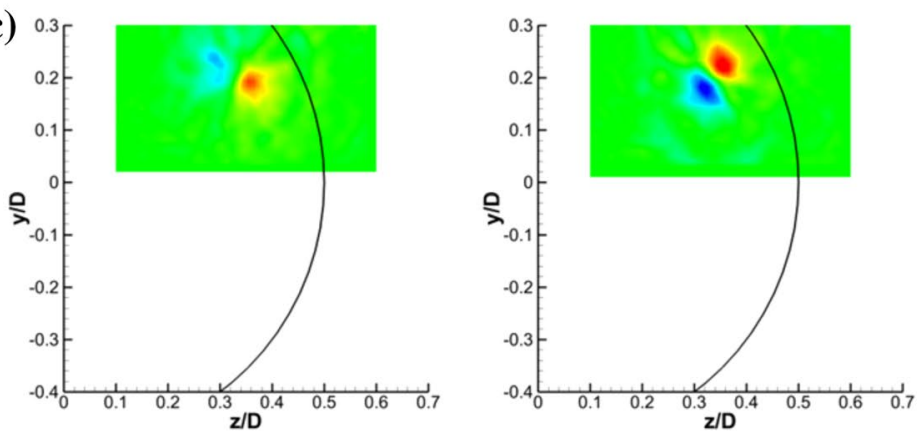

(d)
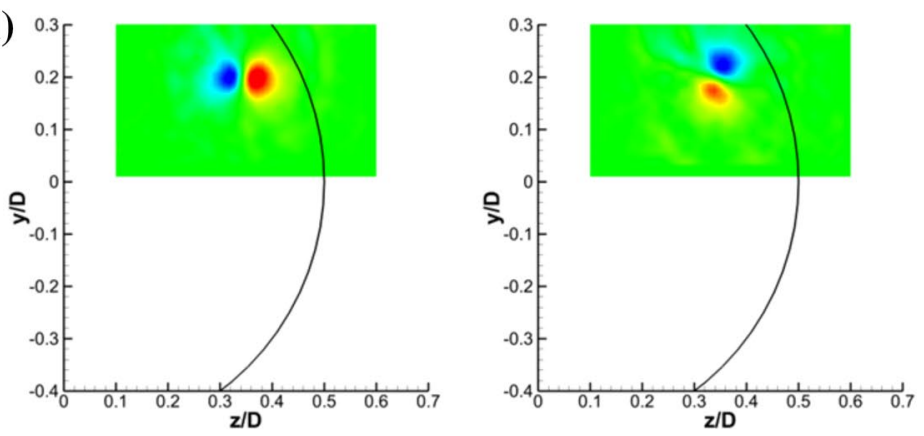

(e)

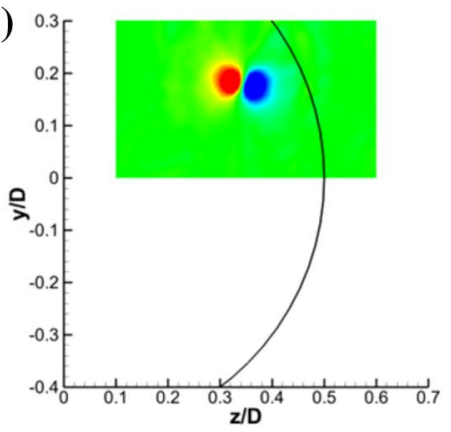


POD modes for the two most extreme upsweep angles $\Phi=24^{\circ}$ and $32^{\circ}$ are shown in Figs. 18 and 19, respectively. The first POD mode at $x / L=0.2$ in Fig. 18a (most upstream measurement plane) closely resembles the timeaveraged flow, suggesting that this corresponds to the pulsations of vortex strength. At further downstream stations, the flow behavior changes drastically as the axisymmetric time-averaged vortex is starting to form. Beyond the second measurement plane $(x / L=0.4)$, two opposite regions of vorticity exist within both POD modes, which gradually form an almost circular vortex dipole towards the trailingedge at $x / L=1.0$. This vortex dipole is centered around the time-averaged core location and is interpreted as a helical displacement of the vortex with an azimuthal wavenumber $m=1$. The physical interpretation of this vortex dipole is such that it represents a decrease in one half of the timeaveraged core vorticity and an increase in the other, resulting in an overall helical displacement of the core (Fabre et al. 2006). It is worthy to note that similar helical displacement modes were previously observed with wing tip vortices (Del Pino et al. 2011; Chen et al. 2016), delta wing vortices (Zhang et al. 2016), and inlet vortices (Wang and Gursul 2012). The helical mode instability was predicted for the Lamb-Oseen vortex (with no axial flow) by considering the transient evolution of flow disturbances (Antkowiak and Brancher 2004) as a function of time. Recently, Edstrand et al. (2016) performed a spatial stability analysis of a Batchelor vortex and found the same $m=1$ helical mode as in their velocity measurements of a tip vortex.

It can be seen that the first and second POD modes at $x / L=1.0$ are nearly orthogonal to each other (Fig. 18e), a linear combination of the two modes can be thought to form displacements of the vortex core which results in its meandering. The POD modes for the largest upsweep angle $\Phi=32^{\circ}$ shown in Fig. 19 exhibit similar mode shapes; however, the modes appear better defined and more coherent at each measurement station, suggesting that the helical mode has become stronger and more dominant with increasing upsweep angle. If vortex meandering is defined as the deviations of the location of the instantaneous vortex center from the center of the time-averaged vortex, only the $|m|=1$ helical mode can represent the meandering for the wave-like disturbances. This is the only mode with nonzero radial velocity at the vortex axis. However, this mode can have different wavelengths (or frequencies). Hence, meandering can be considered to be due to the sum of various wavelengths of the first helical mode. In this study, the frequency information cannot be obtained from the PIV images that are not time-accurate; however, previous investigations (see Wang and Gursul 2012) point out that dominant mode has a very low frequency (or long wavelength, on the order of $10^{2}-10^{3}$ vortex radius).

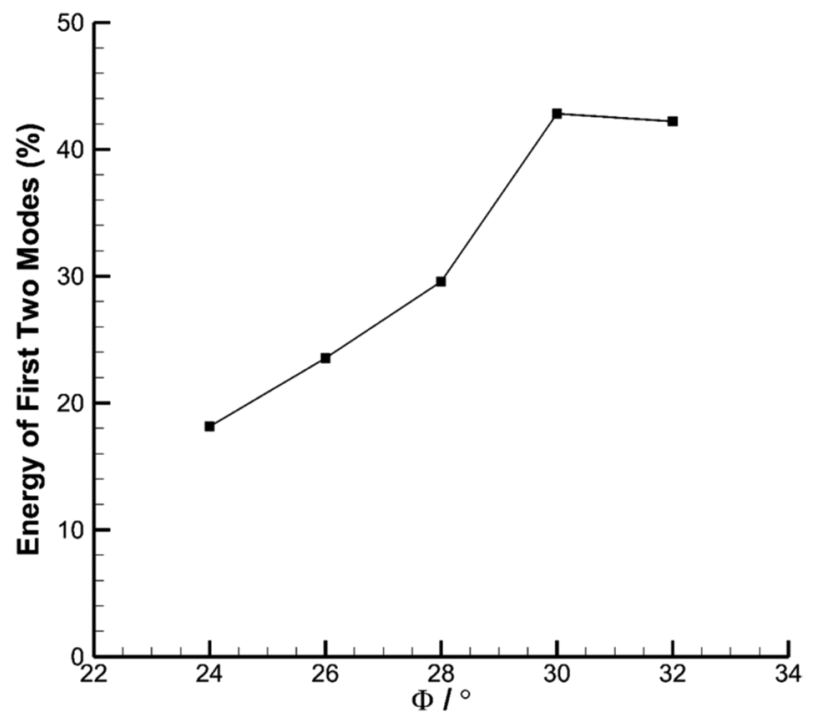

Fig. 21 Percentage energy of the first two modes at $x / L=1.0$ measurement plane as a function of upsweep angle. $R e_{D}=2 \times 10^{5}$

Figure 20 presents a comparison of the effect of upsweep angle on the first two modes of the fully developed afterbody vortex at $x / L=1.0$. The vortex dipole gradually becomes more circular and well defined as $\Phi$ is increased, and are all centered around the location of the time-averaged vortex core. Observing Fig. 20 suggests that the helical mode generally becomes more dominant as $\Phi$ is increased. The sum of the energy of the first two modes at $x / L=1.0$ is presented in Fig. 21 to quantify the effect of the upsweep angle. It is clear that, as $\Phi$ is increased, there has been a large increase in the contribution of mode 1 and mode 2 energies between $\Phi=24^{\circ}$ and $30^{\circ}$ increasing from 18 to $44 \%$, confirming that the dominant wavelength of meandering does indeed become stronger. Beyond $\Phi=30^{\circ}$, the sum of the two mode energies seem to have reached a saturation with only a slight difference in value between $\Phi=30^{\circ}$ and $32^{\circ}$.

\subsection{Vortex pair interactions}

One advantage of the water tunnel data was that we were able to capture both vortices of the vortex pair. Although the time-averaged vortex locations appear perfectly symmetric about the vertical coordinate axis (Fig. 22a), the two instantaneous vortices exhibit asymmetries. There is evidence that the position of one vortex relative to the other deviates significantly, with two extreme cases presented in Fig. 22b, c. The time-averaged flow field smooths the asymmetries and presents a symmetric vortex pair. It is not clear how the instantaneous "symmetry breaking" is caused. Various vortex pair instabilities were considered 
(a)

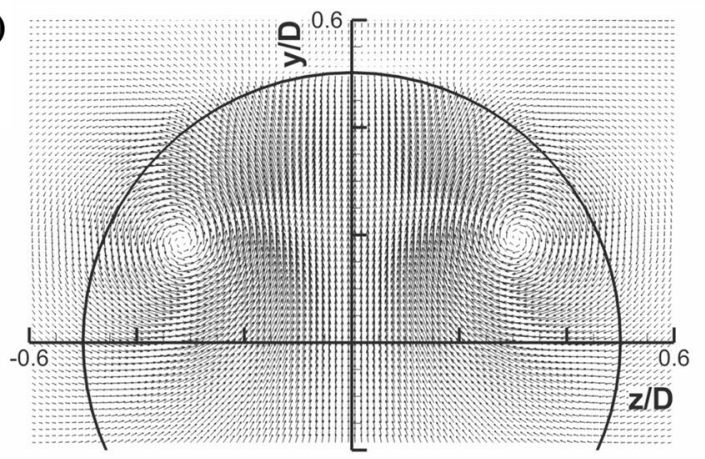

(b)

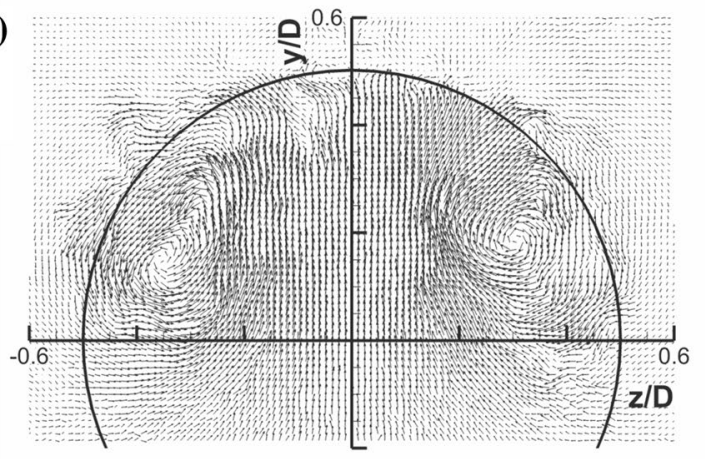

(c)

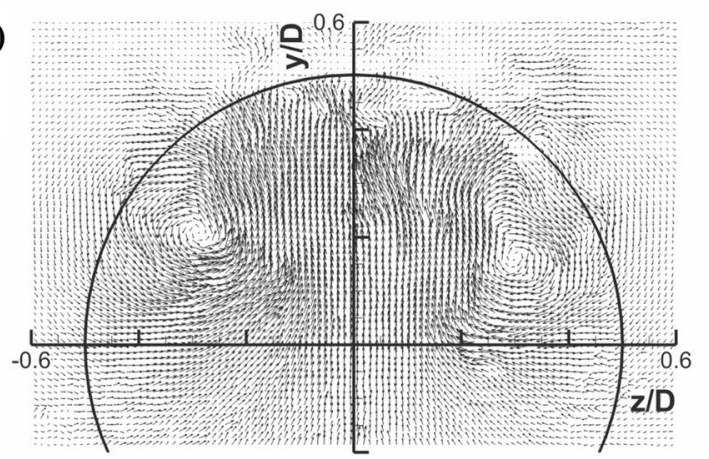

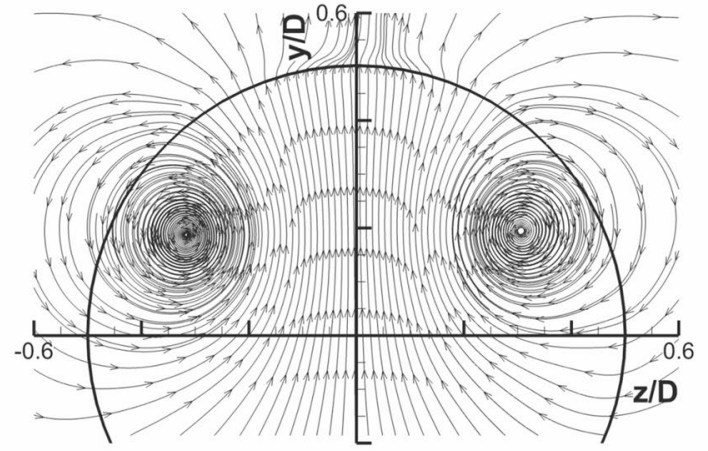
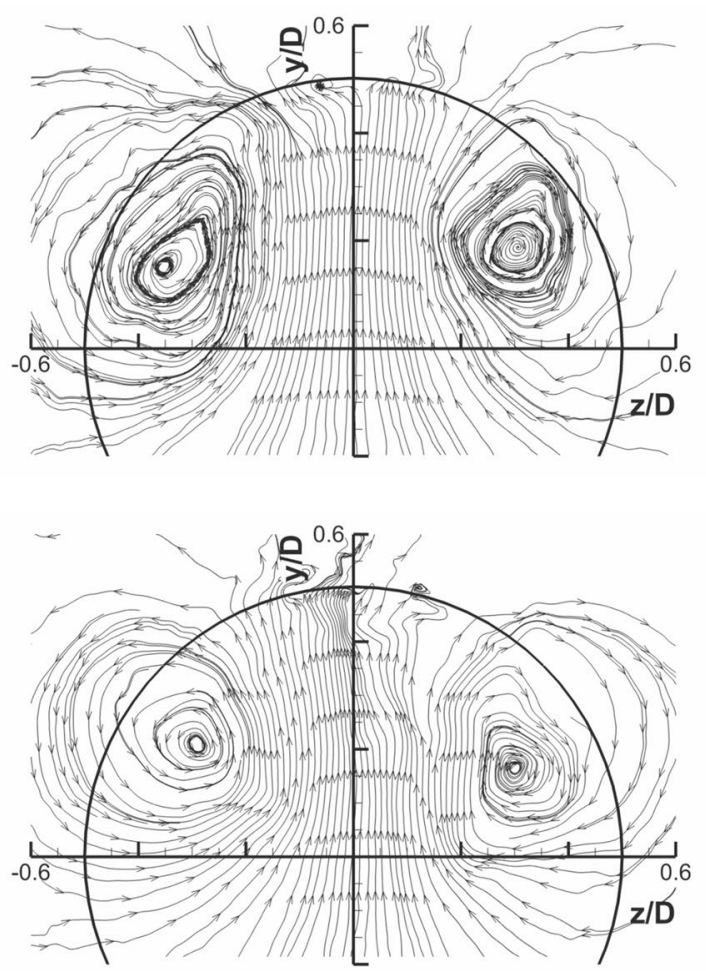

Fig. 22 Vortex pair, velocity vectors (left),streamlines (right). $R e_{D}=2 \times 10^{4}$. a Time-averaged; $\mathbf{b}$ and $\mathbf{c}$ two examples of asymmetry in instantaneous vortex location

as potential causes. The ratio of the vortex core radius $r_{\text {core }}$ to the vortex separation distance $b$ is around $r_{\text {core }} / b \approx 0.2$. According to Leweke et al. (2016), both long wavelength and short wavelength instabilities can be amplified for this aspect ratio. To investigate possible time-dependent interactions of the vortex pair, instantaneous vortex locations in the crossflow plane were analyzed. As before, the instantaneous vortex centers were defined by the maximum value of the $Q$ criterion. The correlation coefficient between the $y$ coordinate of each vortex was first calculated, before finding the equivalent for the $z$ coordinate. The variation of vortex correlation in the streamwise direction is shown in Fig. 23 for the vertical and horizontal directions separately. The magnitude of the correlation coefficient, $R$, is consistently below $|R| \leq 0.1$ at all streamwise stations, indicating that there is no appreciable correlation between the two vortex positions. This could be due to the weak short wavelength instabilities and insufficient streamwise distance for the long wavelength instabilities to develop.

\section{Conclusions}

The development of afterbody vortices on a generic cylindrical body with a slanted base has been studied experimentally in a wind tunnel at $R e_{D}=2 \times 10^{5}$ and a water tunnel at $R e_{D}=2 \times 10^{4}$. Upsweep angles in the range of $\Phi=24^{\circ}-32^{\circ}$ were considered in the wind 


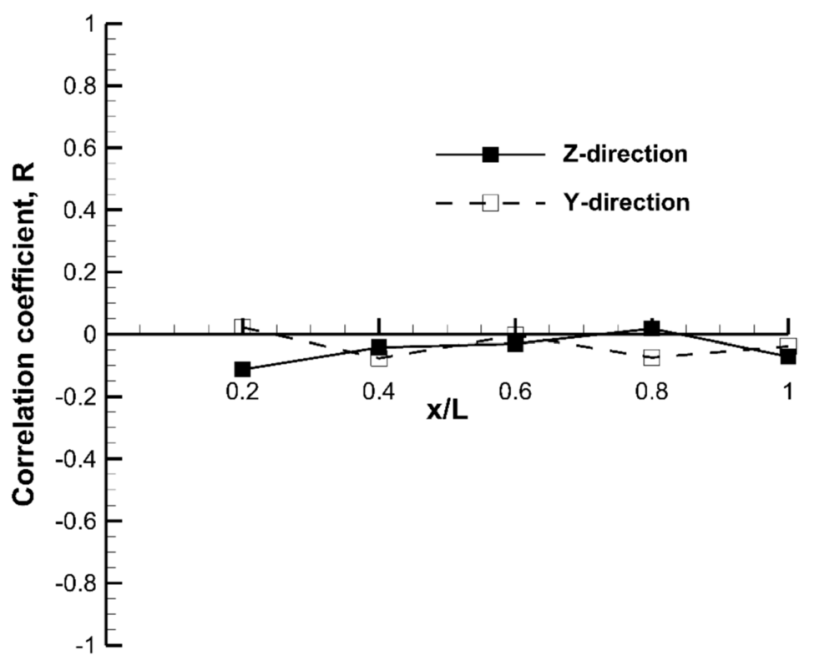

Fig. 23 Variation of correlation coefficient of instantaneous vortex locations, $R_{D}=2 \times 10^{4}$

tunnel experiments. Force measurements revealed an increase in drag coefficient of about $50 \%$ across the range of upsweep angles considered, and showed good agreement with previous studies. The vortex circulation at the trailing-edge was proportional to the drag coefficient, and resulted in an increase of about $50 \%$ across the range of upsweep angles tested. The initial vortex formation process by means of vorticity shedding from the separated shear layer and subsequent roll-up appeared to be complete at around $x / L=0.6$, after which the rolledup vortex progressively moved away from the surface. Correspondingly, the vortex footprint on surface pressure resulting in the largest suction was observed when the vorticity concentration was closer to the surface, for approximately $x / L \leq 0.4$. Towards the trailing-edge the vortices became more axisymmetric and grew in size with increasing upsweep angle. For one particular upsweep angle, $\Phi=28^{\circ}$, the effect of the Reynolds number on the afterbody vortices was investigated. It was found that the vortex roll-up is delayed for the lower Reynolds number, and the resulting vortex at the trailing-edge was slightly stronger and caused a slightly larger drag coefficient.

Analysis of the instantaneous flow fields revealed that, for all upsweep angles, the vortex evolved to become more coherent towards the trailing-edge, with reduced meandering which resulted in a smaller time-averaged core radius. This decrease of meandering amplitude and time-averaged core radius in the streamwise direction was believed to be the first documented example in external aerodynamic vortex flows. The onset of the reduction in meandering amplitude was initiated further upstream with increasing upsweep angle. Proper Orthogonal Decomposition revealed the helical displacement mode, with azimuthal wavenumber $m=1$, as the dominant mode for the fully developed vortex towards the trailingedge. This same helical displacement mode was previously observed with wing tip and delta wing vortices. The contribution of the first two modes to the total energy increased from 18 to $44 \%$ within increasing upsweep angles tested. Although the time-averaged vortex pair is symmetric, the instantaneous vortex pair exhibits asymmetry. However, very low correlation exists between the displacements of the vortex centers, suggesting weak vortex pair instabilities.

Acknowledgements This work was supported by the Air Force Office of Scientific Research, Air Force Material Command, USAF, under Grant Number FA9550-14-1-0126.

Open Access This article is distributed under the terms of the Creative Commons Attribution 4.0 International License (http://creativecommons.org/licenses/by/4.0/), which permits unrestricted use, distribution, and reproduction in any medium, provided you give appropriate credit to the original author(s) and the source, provide a link to the Creative Commons license, and indicate if changes were made.

\section{References}

Adrian RJ, Christensen KT, Liu ZC (2000) Analysis and interpretation of instantaneous turbulent velocity fields. Exp Fluids 29:275-290. doi:10.1007/s003489900087

Antkowiak A, Brancher P (2004) Transient energy growth for the Lamb-Oseen vortex. Phys Fluids 16:1-4. doi:10.1063/1.1626123

Bearman PW (1980) Review-Bluff body flows applicable to vehicle aerodynamics. J Fluids Eng T ASME 102:265-274. doi:10.1115/1.3240679

Berkooz G, Holmes P, Lumley JL (1993) The proper orthogonal decomposition in the analysis of turbulent flows. Annu Rev Fluid Mech 25:539-575. doi:10.1146/annurev.fl.25.010193.002543

Britcher CP, Alcorn CW (1991) Interference-free measurements of the subsonic aerodynamics of slanted -base ogive cylinders. AIAA J 29:520-525. doi:10.2514/3.10614

Bury Y, Jardin T, Klockner A (2013) Experimental investigation of the vortical activity in the close wake of a simplified military transport aircraft. Exp Fluids 54:1524. doi:10.1007/ s00348-013-1524-4

Calarese W, Crisler WP, Gustafson GL (1985) Afterbody drag reduction by vortex generators. In: AIAA-1985-0354, 23rd AIAA aerospace sciences meeting, Reno, NV, USA doi: 10.2514/6.1985-354

Chen H, Reuss DL, Sick V (2012) On the use and interpretation of proper orthogonal decomposition of in-cylinder engine flows. Meas Sci Technol 23:085302. doi:10.1088/0957-0233/23/8/085302

Chen H, Hung DLS, Reuss DL, Sick V (2013) A practical guide for using proper orthogonal decomposition in engine research. Int $\mathrm{J}$ Engine Res 14:307-319. doi:10.1177/1468087412455748

Chen C, Wang Z, Cleaver DJ, Gursul I (2016) Interaction of trailing vortices with downstream wings. In: AIAA-2016-1848, 54th AIAA aerospace sciences meeting, San Diego, CA, USA doi: 10.2514/6.2016-1848

Del Pino C, Lopez-Alonso JM, Parras L, Fernandez-Feria R (2011) Dynamics of the wing-tip vortex in the near field of a NACA 0012 aerofoil. Aeronaut J 115:229-239. doi:10.1017/ S0001924000005686 
Devenport WJ, Rife MC, Liapis SI, Follin GJ (1996) The structure and development of a wing-tip vortex. J Fluid Mech 312:67106. doi:10.1017/S0022112096001929

Edstrand AM, Davis TB, Schmid PJ, Taira K, Cattafesta LN (2016) On the mechanism of trailing vortex wandering. J Fluid Mech 801:R1-1-R1-11. doi:10.1017/jfm.2016.440

Epstein RJ, Carbonaro MC, Caudron F (1994) Experimental investigation of the flowfield about an upswept afterbody. J Aircr 31:1281-1290. doi:10.2514/3.46648

Fabre D, Sipp D, Jacquin L (2006) Kelvin waves and the singular modes of the Lamb-Oseen vortex. J Fluid Mech 551:235-274. doi: $10.1017 / \mathrm{S} 0022112005008463$

Graftieaux L, Michard M, Nathalie G (2001) Combining PIV, POD and vortex identification algorithms for the study of unsteady turbulent swirling flows. Meas Sci Technol 12:1422-1429. doi:10.1088/0957-0233/12/9/307

Hunt JCR, Wray AA, Moin P (1988) Eddies, stream and convergence zones in turbulent flows. Center for Turbulence Research Report CTR-S88 No. 193

Jackson R, Wang Z, Gursul I (2015) Control of afterbody vortices by blowing. In: AIAA-2015-2777, 45th AIAA fluid dynamics conference, Dallas, TX, USA doi: 10.2514/6.2015-2777

Jeong J, Hussain F (1995) On the identification of a vortex. J Fluid Mech 285:69-94. doi:10.1017/S0022112095000462

Leweke T, Le Dizés S, Williamson CHK (2016) Dynamics and instabilities of vortex pairs. Annu Rev Fluid Mech 48:507-541

Margaris P, Marles D, Gursul I (2008) Experiments on jet/vortex interaction. Exp Fluids 44:261-278. doi:10.1007/s00348-007-0399-7

Maull DJ (1980) The drag of slant-based bodies of revolution. Aeronaut J 84:164-166

McCluney B, Marshall J (1967) Drag development of Belfast. Aircr Eng 39:33-37. doi:10.1108/eb034302
Menke M, Gursul I (1997) Unsteady nature of leading edge vortices. Phys Fluids 9:2960-2966. doi:10.1063/1.869407

Moffat RJ (1988) Describing the uncertainties in experimental results. Exp Therm Fluid Sci 1:3-17. doi:10.1016/0894-1777(88)90043-X

Morel T (1980) Effect of base slant on flow in the near wake of an axisymmetric cylinder. Aeronaut Quart 31:132-147

Peake DJ, Rainbird WJ, Atraghji EG (1972) Three-dimensional flow separations on aircraft and missiles. AIAA J 10:567-580. doi: $10.2514 / 3.50159$

Smith BR, Yagle PJ, Hooker JR (2013) Reduction of aft fuselage drag on the C-130 using microvanes. In: AIAA-2013-0105, 51st AIAA aerospace sciences meeting, Grapevine, TX, USA doi:10.2514/6.2013-105

Stuart AD, Jones AT (1977) The drag of an upswept rear fuselage. Undergraduate Project, Imperial College London, London

Telli H, Ayan E, Soyer S, Gülsever E and Özcan S (2016) An investigation of C-130 aircraft base drag reduction with afterbody modifications. In: AIAA-2016-3430, 34th AIAA applied aerodynamics conference, Washington, DC, USA doi:10.2514/6.2016-3430

Wang Z, Gursul I (2012) Unsteady characteristics of inlet vortices. Exp Fluids 53:1015-1032. doi:10.1007/s00348-012-1340-2

Wortman A (1999) Reduction of fuselage form drag by vortex flows. J Aircr 36:501-506. doi:10.2514/2.2484

Xia XJ, Bearman PW (1983) Experimental investigation of the wake of an axisymmetric body with a slanted base. Aeronaut Quart 34:24-45

Zhang X, Wang Z, Gursul I (2016) Interaction of multiple vortices over a double delta wing. Aerosp Sci Technol 48:291-307. doi:10.1016/j.ast.2015.11.020 